Materials and Components Technology Division Materials and Components Technology Division Materials and Comporients Technology Division

\section{Chaotic Vibrations of Nonlinearly Supported Tubes in Crossflow}

by Y. Cai and S. S. Chen

Argonne National Laboratory, Argonne, Illinois 60439

operated by The University of Chicago

for the United States Department of Energy under Contract W-31-109-Eng-38 
Argonne National Laboratory, with facilities in the states of Illinois and Idaho, is owned by the United States government, and operated by The University of Chicago under the provisions of a contract with the Department of Energy.

\section{DISCLAIMER}

This report was prepared as an account of work sponsored by an agency of the United States Government. Neither the United States Government nor any agency thereof, nor any of their employees, makes any warranty, express or implied, or assumes any legal liability or responsibility for the accuracy, completeness, or usefulness of any information, apparatus, product, or process disclosed, or represents that its use would not infringe privately owned rights. Reference herein to any specific commercial product, process, or service by trade name, trademark, manufacturer, or otherwise, does not necessarily constitute or imply its endorsement, recommendation, or favoring by the United States Government or any agency thereof'. The views and opinions of atuthors expressed herein do not necessarily state or reflect those of the United States Government or any agency thereof.

Reproduced from the best available copy.

Available to DOE and DOE contractors from the Office of Scientific and Technical Information

$$
\text { P.O. Box } 62
$$

Oak Ridgc, TN 378.31

Prices available from (61.5) $576-8401$

Available to the public from the

National Technical Information Service

U.S. Department of Commere

528.5 Porl Royal Road

Springlicld, VA 22161 
Chaotic Vibrations of Nonlinearly Supported Tubes DE92 014074 in Crossflow

by

Y. Cai and S. S. Chen

Materials and Components Technology Division

February 1992

Work supported by

U.S. DEPARTMENT OF ENERGY

Office of Basic Energy Sciences 


\section{Contents}

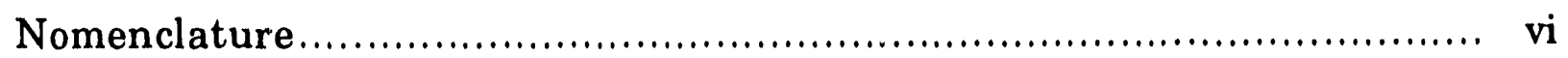

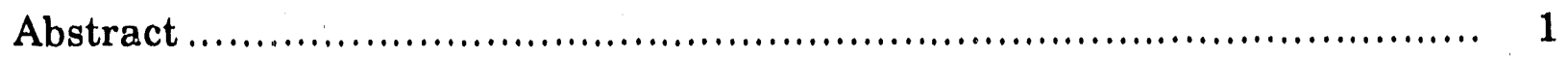

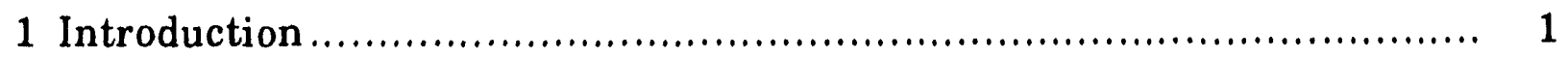

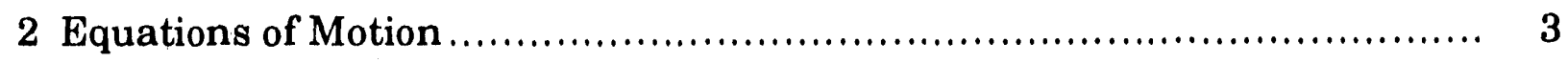

3 Numerical Simulations ...................................................... 5

4 Measuring Chaotic Motion................................................. 6

4.1 Phase Flow Portraits............................................................. 6

4.2 Power Spectral Density .................................................... 8

4.3 Poincaré Map............................................................ 9

4.4 Lyapunov Exponent ........................................................ 10

4.5 Fractal Dimensions .................................................. 12

4.6 Bifurcation Diagram......................................................... 17

4.7 Measurements with Asymmetric Gaps at the TSP ................... 22

5 Conclusions...................................................................... 23

Acknowledgments........................................................ 26

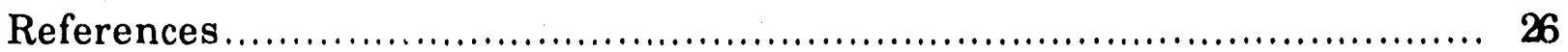

Appendix: Modal Analysis of a Bilinear Model................................... 29 


\section{Figures}

1 Schematic representation of a tube and supports in crossflow.......... 4

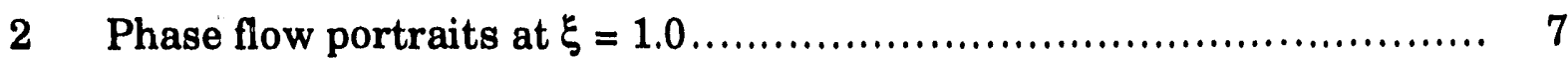

$3 \quad$ Power spectral density of tube displacement ......................... 8

4 Sampling trigger signal for Poincaré map................................ 10

$5 \quad$ Poincaré map of tube motion ......................................... 11

6 Geometric interpretation of Poincare sections in the threedimensional phase space............................................... i2

$7 \quad$ Poincaré map of tube motion............................................ 13

8 Lyapunov exponents of tube motion..................................... 15

9 Correlation function and information entropy for set of points in Poincaré map in Fig. 5b............................................... 16

10 Correlation dimension: local slope of correlation function in Fig. 9... 16

11 Bifurcation diagram of tube motion in instability region................. 18

12 System damping ratio vs. mean flow velocity, Model 1 and Model $2 \ldots . .19$

13 PSD, Poincaré map, and Lyapunov exponent of tube motion with $\mathrm{U}_{\mathrm{m}}=4.6 \mathrm{~m} / \mathrm{s}$

14 PSD, Poincaré map, and Lyapunov exponent of tube motion with

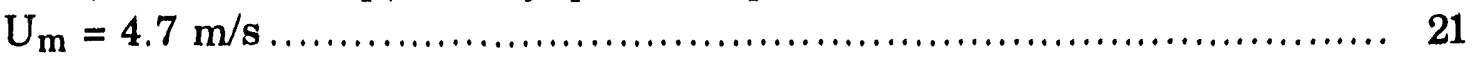

15 Bifurcation diagrams of tube motion.................................. 23

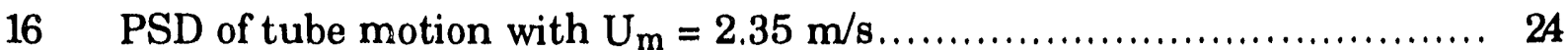

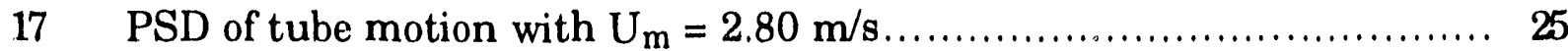




\section{Table}

A1 Eigenvalues $k_{\text {in }}$ of first 10 modes for Models 1 and $2 \ldots \ldots \ldots \ldots \ldots \ldots \ldots . \ldots . \ldots 32$ 


\section{Nomenclatiure}

$a_{\text {in }}, \dot{a}_{\text {in }} \quad$ Generalized tube displacement and velocity for Model i

C Correlation function

D Tube diameter

$\mathrm{d}_{\mathrm{G}} \quad$ Correlation dimension

$\mathrm{d}_{\mathrm{I}} \quad$ Information dimension

E Young's modulus

$e_{1}, e_{2} \quad$ Tube-support gaps

f Oscillation frequency

$f_{n} \quad$ Natural frequency of $n$-th mode

I Mcment of inertia of tube cross section

I(r) Information entropy

$\mathrm{K}_{\mathrm{c}} \quad$ Equivalent stiffness

$\mathbf{k}_{\text {in }} \quad$ Eigenvalues for tube vibration of Model $\mathbf{i}$

$\ell \quad$ Tube length

m Tube mass per unit length

$\mathrm{N} \quad$ Number of total points in Poincaré maps

n Number of modes

P Pitch

$P_{i} \quad$ Probability in i-th cell on Poincaré set

R Radius of tube

r Sphere length

t Time 
$t_{s} \quad$ Time when tube strikes TSP

$t_{d} \quad$ Time when tube leaves TSP

$\mathrm{U}_{\mathrm{m}} \quad$ Mean flow velocity

$\mathrm{U}_{\mathrm{r}} \quad$ Reduced flow velocity $\left(=\mathrm{U}_{\mathrm{m}} / \mathrm{fD}\right.$ or $\left.\mathrm{U} / \mathrm{fD}\right)$

$\mathrm{u}_{\mathrm{i}}, \dot{\mathrm{u}}_{\mathrm{j}} \quad$ Displacement and velocity

$\mathbf{x}_{\mathbf{i}} \quad$ Points in phase space of Poincaré map

$\alpha_{\text {in }} \quad$ Added-mass coefficients of $n$-th mode for Model $i$

$\alpha_{i n}^{d} \quad$ Fluid damping coefficients of $n$-th mode for Model $\mathbf{i}$

$\alpha_{i n}^{e} \quad$ Fluid stiffness coefficients of $n$-th mode for Model i

$\zeta_{\mathrm{n}} \quad$ Damping ratio in vacuum

$\gamma \quad$ Mass ratio $\left(=\rho \pi R^{2} / \mathrm{m}\right)$

$\xi \quad$ Dimensionless coordinate

$\rho \quad$ Fluid density

$\varphi_{\text {in }}(\xi) \quad$ Orthonormal function of $\mathrm{n}$-th mode for Model $\mathbf{i}$

$\phi_{0} \quad$ Phase angle

$\omega_{\mathrm{i}} \quad$ Circular frequency for Model $\mathrm{i}$

$\omega_{\text {in }} \quad$ Natural frequency in radian of tube in vacuum

\section{Subscripts}

i $\quad \mathrm{i}=1,2$ for Model 1 and 2, respectively

n n-th modes

1 For Model 1 (TSP-inactive mode)

$2 \quad$ For Model 2 (TSP-active mode) 


\title{
Chaotic Vibrations of Nonlinearly Supported Tubes in Crossflow
}

\author{
by
}

Y. Cai and S. S. Chen

\begin{abstract}
By means of the unsteady-flow theory and a bilinear mathematical model, a theoretical study is presented for chaotic vibrations associated with the fluidelastic instability of nonlinearly supported tubes in a crossflow. Effective tools, including phase portraits, power spectral density, Poincaré maps, Lyapunov exponent, fractal dimension, and bifurcation diagrams, are utilized to distinguish periodic and chaotic motions when the tubes vibrate in the instability region. The results show periodic and chaotic motions in the region corresponding to fluid-damping-controlled instability. Nonlinear supports, with symmetric or asymmetric gaps, significantly affect the distribution of periodic, quasiperiodic, and chaotic motions of a tube exposed to various flow velocities in the instability region of the tube-support-plate-inactive mode.
\end{abstract}

\section{Introduction}

Extensive experimental and analytical studies have been performed on the dynamic response of loosely hald tubes and how the tube response is related to wear (Chen, 1991; Cai et al., 1991). Chen et al. (1984) investigated the fluidelastic behavior of loosely held tubes in the laboratory. They observed that as the flow velocity is increased to a threshold value, or critical flow velocity, instability in the tube-support-plate (TSP)-inactive mode may occur. Then, for a range of flow velocities higher than the threshold flow velocity, the tube vibrates predominantly in the TSP-inactive mode, with the response amplitude limited by the clearance between the tube and the TSP. With a further increase in flow velocity, a second threshold is reached and instability in the TSP-active mode begins. In this range, large-amplitude oscillations occur and, in many cases, tubes may strike one another. Additional experimental studies to determine the response of loosely supported tubes in the TSP-inactive mode under specific flow conditions have recently been published (Nakamura and Fujita, 1987; Fisher and Ingham, 1988; Antunes et al., 1991). 
For the analysis of tube responses and impacting behavior of loosely supported tubes, many nonlinear methods have been developed in recent years. Numerical simulations were performed by Axisa et al. (1988), Fricker (1988), and Rao et al. (1988); all used quasistatic- or quasisteady-flow theories, which are applicable in specific parameter ranges. Chen and Chandra (1990) developed the unsteady-flow model for fluidelastic instability of tubes in nonuniform flow. Cai et al. (1991) presented a bilinear model based on the unsteady-flow theory. The simulations by Cai et al. (1991) agreed reasonably well with the experimental data of Chen et al. (1984) and demonstrated that the unsteady-flow theory and the bilinear model are adequate to describe the nonlinear behavior of fluidelastic instability associated with TSP-inactive modes of loosely supported tubes in crossflow.

Bccause of the recent interest in chaotic motions of nonlinear systems (Moon, 1987) and the relationship of chaotic vibration to tube wear, it is appropriate to look into the possible existence of chaos in tube arrays in crossflow. Related systems without flow have been studied experimentally by several investigators. Moon (1983), Shaw and Holmes (1983), and Shaw (1985a, 1985b) studied forced oscillation of beams with motion constraints; chaos was found. A case study of chaos in a marine application that involved impacting modeled by bilinear springs was discussed by Thompson and Stewart (1986). Recently, it was realized that flowinduced vibration of loosely supported tubes, which is one of the systems with motion constraints, can display a wide variety of dynamic behaviors. For example, chaotic fluidelastic vibrations of a constrained, fluid-conveying pipe were examined by Paidoussis and Moon (1988 and 1989), both experimentally and theoretically, with a two-degree-of-freedom system. A study of chaotic and periodic motion of a nonlinear oscillator used to model flow-induced vibration of loosely supported tubes was conducted by Langre et al. (1990).

Based on the unsteady-flow theory and a bilinear model, an analytical study of the dynamics of loosely supported tubes in crossflow was performed by Cai and Chen (1991). Many computations were conducted to confirm the existence of chaotic motion and the route to chaos with the change of control parameters in the instability region of the TSP-inactive mode. Indeed, it was demonstrated through the use of several effective techniques, including bifurcation diagrams, phase flow portraits, power spectral density, and Poincaré maps, that chaotic motion was possible for such an autonomous system. Also, a preliminary test was conducted to obtain convincing evidence of periodic/chaotic dynamics. The results of the test qualitatively agree with analytical results based on the response spectral densities and observation.

The purpose of this report is to apply the mathematical models and other techniques used in the earlier work of the authors (Cai et al., 1991; Cai and Chen, 
1991) to extensively analyze the periodic/chaotic dynamics of a tube nonlinearly supported in crossflow. The interesting regions of periodic and chaotic motion were expanded to the whole region of fluidelastic instability of tubes associated with velocity-controlled negative damping between two threshold values of flow velocity. A route of chaotic-periodic-chaotic motions was predicted when values of negative damping vary with mean flow velocity in this region. Some new measuring tools, e.g., the Lyapunov exponent and fractal dimensions, were added to others such as the phase portrait, power spectral density, and Poincaré maps, for distinguishing periodic/chaotic motions. Indeed, those measuring tools proved quite effective in predicting the existence of periodic and chaotic motion in the instability region. Tube response characteristics with various symmetric or asymmetric gap conditions were also studied. The nonlinearity of the symmetric

or asymmetric gaps significantly affects the distribution of periodic, quasiperiodic, and chaotic motions of tubes exposed to various flow velocities in the instability region of the TSP-inactive mode.

\section{Equations of Motion}

An unsteady-flow theory for fluidelastic instability of a row of tubes in crossflow was described in detail by Chen $(1983,1989)$ and by Chen and Chandra (1990). A bilinear mathematical model for loosely supported tubes vibrating in crossflow was well defined by Cai et al. (1991). For completeness, the unsteadyflow model for fluidelastic instability of loosely supported tubes is briefly described here. Readers interested in further details of these theories can refer to our previous work.

Consider the case of fluidelastic instability in which a velocity-controlled negative damping mechanism is dominant. The stability of the tube row may be analyzed approximately by considering only one flexible tube among other rigid tubes and neglecting the coupling in the two directions. Therefore, a schematic representation of a two-span flexible tube with one intermediate support, vibrating in one direction, is shown in Fig. 1. When the right end (C3) of the tube does not strike the stop, it is a pinned-pinned-free model (Model 1). When the right end strikes the stop, it becomes a pinned-pinned-spring-supported model (Model 2). Because the effects of impact force are represented by the linear springs at $\mathrm{C} 3$ in Model 2, the vibratory system is autonomous. Caps $e_{1}$ and $e_{2}$ at C3 provide nonlinear support for the system, which may cause chaotic vibrations when some system parameters vary within certain ranges.

The solutions of tube vibration by the normal-mode method can be described as two models analyzed in two different time regions (Cai et al., 1991): 


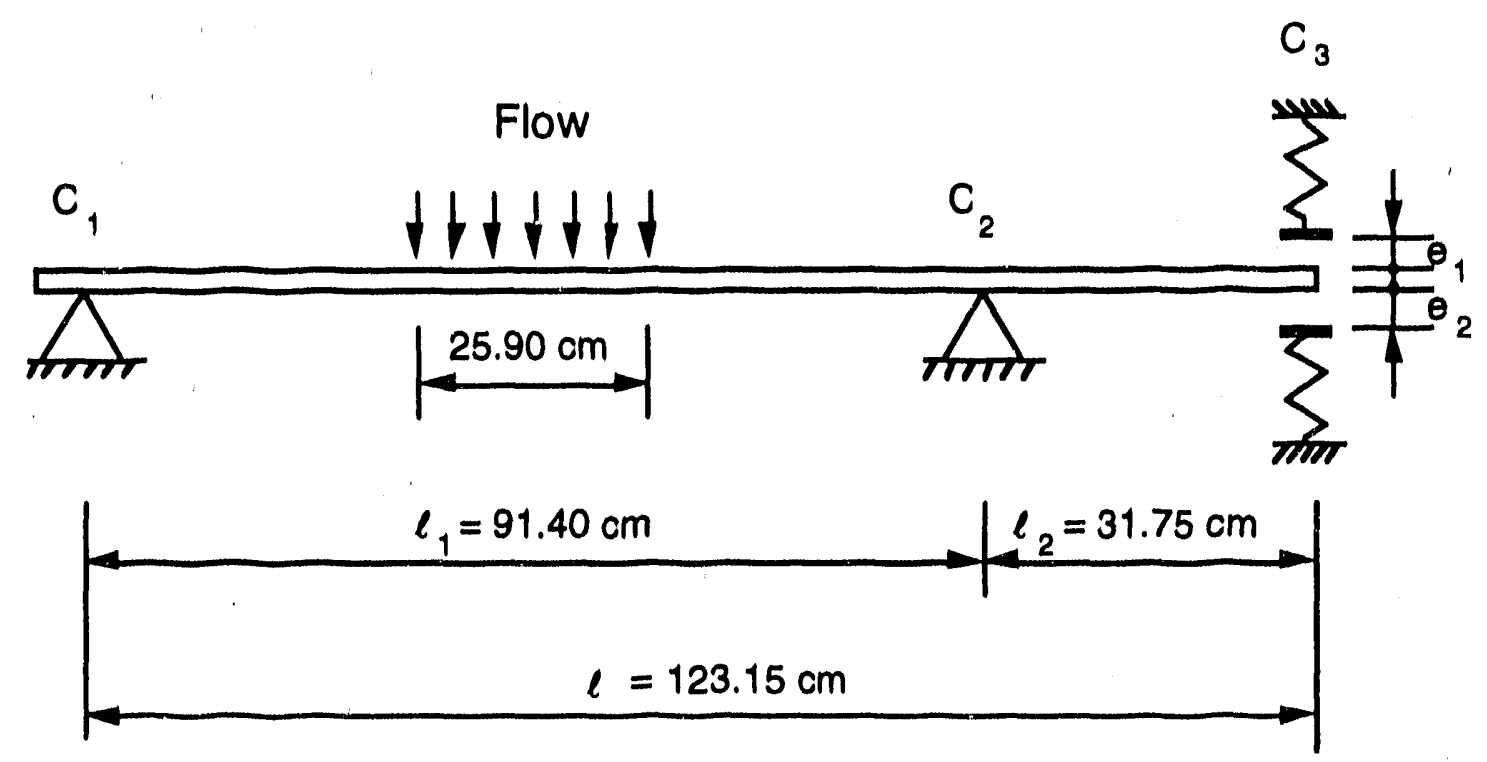

Fig. 1. Schematic representation of a tube and supports in crossflow

$$
\begin{aligned}
& u(\xi, t)=\sum_{n=1}^{\infty} a_{1 n}(t) \varphi_{1 n}(\xi) \\
& 0<\mathrm{t}<\mathrm{t}_{\mathrm{s}} \quad \text { (Model 1) } \\
& \dot{\mathrm{u}}(\xi, \mathrm{t})=\sum_{\mathrm{n}=1}^{\infty} \dot{\mathrm{a}}_{1 \mathrm{n}}(\mathrm{t}) \varphi_{1 \mathrm{n}}(\xi)
\end{aligned}
$$

and

$$
\left.\begin{array}{l}
u(\xi, t)=\sum_{n=1}^{\infty} a_{2 n}(t) \varphi_{2 n}(\xi)+\left.u(\xi, t)\right|_{t=t_{s}} \\
\dot{u}(\xi, t)=\sum_{n=1}^{\infty} \dot{a}_{2 n}(t) \varphi_{2 n}(\xi)
\end{array}\right\} t_{s}<t<t_{d} \text { (Model 2) }
$$

where $\mathrm{u}(\xi, \mathrm{t})$ and $\dot{\mathrm{u}}(\xi, \mathrm{t})$ are tube displacement and velocity, respectively; $\varphi_{1 \mathrm{n}}(\xi)$ and $\varphi_{2 n}(\xi)$ are the normal modes of Models 1 and 2 (see Appendix); $\xi=z / \ell ; t_{\mathrm{s}}$ is the time when the right end of the tube strikes the stop, $t_{d}$ is the time when the tube end leaves the stop; and $a_{1 n}(t)$ and $a_{2 n}(t)$ are the solutions of the equations 
$\left(1+\gamma \alpha_{i n}\right) \frac{d a_{i n}^{2}}{d t^{2}}+\left(2 \zeta_{n} \omega_{i n}-\frac{\gamma U_{r}^{2}}{\pi^{3}} \omega_{i} \alpha_{i n}^{d}\right) \frac{d a_{i n}}{d t}+\left(\omega_{i n}^{2}-\frac{\gamma U_{r}^{2}}{\pi^{3}}-\alpha_{i n}^{e}\right) a_{i n}=0$

$i=1$ and 2 for Models 1 and 2 , respectively, and

$\mathrm{n}=1,2,3, \infty_{\infty}$.

In Eq. 3,

$$
\begin{aligned}
& \gamma=\frac{\rho \pi R^{2}}{m}, \\
& U_{r}=\frac{\pi U_{m}}{\omega_{i} R},
\end{aligned}
$$

and

$$
\omega_{i}=\sqrt{\frac{\omega_{\text {in }}^{2}-\frac{\gamma U_{r}^{2}}{\pi^{3}} \alpha_{\text {in }}^{e}}{1+\gamma \alpha_{i n}}}
$$

Note that $R$ is tube radius; $\rho$ is fluad density; $m$ is tube mass per unit length; $\mathrm{J}_{m}$ is mean nlow velocity; and $\alpha_{i n}, \alpha_{i n}^{\mathrm{d}}$, and $\alpha_{\text {in }}^{\mathrm{e}}$ are added-mass, fluid-damping, and fluid-stiffness coefficients, respectively, and are based on the experimental data of Tanaka (1980) and compiled and evaluated by Chen and Chandra (1990). Notice that $\omega_{i}$ depends on the coefficients $\alpha_{i n}^{e}$, which depend on the reduced flow velocity $U_{r}$ (Eq. 5). Therefore, an iterated method is required to calculate $\omega_{\mathrm{i}}$.

\section{Numerical Simulations}

Numerical simulations of a loosely supported tube, schematically shown in Fig. 1, were carried out with system parameters similar to those applied in previous work (Cai and Chen, 1991). The span between supports $\mathrm{C} 1$ and $\mathrm{C} 2$ is submerged in fluid but subjected to flow at the middle portion only. The tube in Fig. 1 is brass, with a $1.59 \mathrm{~cm}$ outside diameter, $1.59 \mathrm{~mm}$ wall thickness, and $123.15 \mathrm{~cm}$ length. The modulus of elasticity $\mathrm{E}$ is $1.6 \times 10^{6} \mathrm{~kg} / \mathrm{cm}^{2}$. Impact stiffness of springs at the TSP is assumed to be constant, namely, $K_{c}=10^{7} \mathrm{~N} / \mathrm{m}$. Gaps $e_{1}$ and $e_{2}$ are variable control parameters in the simulations and will range from 1.27 to $2.54 \mathrm{~mm}$. 
The computations were carried out on a Sun workstation computer. Before simulations were performed, the natural frequencies of the first 10 modes for both Models 1 and 2 were calculated. Fluid force coefficients $\alpha_{i n}, \alpha_{i n}^{d}$, and $\alpha_{i n}^{e}$ were calculated on the basis of Tanaka's data (1980) for a tube row with a pitch-todiameter ratio of 1.33. The natural frequencies of the first 10 modes of the two models form the basis for determining the time-integration steps required during simulation. As verified in previous studies (Cai et al., 1991; Cai and Chen, 1991), 10 modes (covering a frequency range of $0-1700 \mathrm{~Hz}$ ) give sufficient accuracy for this case. Therefore, 10 modes were used throughout, and the time-integration step for the 10 modes was taken to be $\Delta t=0.0001 \mathrm{~s}$ to ensure the accuracy of simulations. Furthermore, double-precision must be taken throughent the simulations. Numerical integrations were run for a relatively long fixed time to ensure that transient effects had died out before the output was examined. At least 100,000 points were calculated for time histories of tube motions; then the first 4,000 points in the calculated time histories were removed to eliminate transient exfects.

\section{Measuring Chaotic Motion}

As summarized in a previous study (Cai and Chen, 1991), when flow velocity-which has been chosen as the main control parameter-is lower than the critical flow velocity, system damping is positive and tube motion is stable. When flow velocity exceeds the critical flow velocity, the tube loses its stability and begins self-excitation oscillations corresponding to a Hopf bifurcation. If the tube strikes the TSP irregularly in the instability region, tube motion depends on flow velocity and other parameters and chaotic motion may occur. If the tube strikes the TSP regularly after flow velocity exceeds some value in the instability region, tube motion is almost independent of flow velocity and periodic uscillations will occur with a fixed amplitude equal to the diametral clearance.

In this study, the focus was on the instability region, and more measurement techniques than those in the previous study were used to distinguish periodic and chaotic vibration of the tube. Although many computations were performed, only some typical results are presented.

\subsection{Phase Flow Portraits}

First, the time histories of tube motion with symmetric clearance $e_{1}=e_{2}=$ $1.27 \mathrm{~mm}$ at the TSP were calculated, and phase flow diagrams at different flow velocities corresponding to periodic and chaotic motion, shown in Fig. 2, were constructed. In the calculation, the transient was eliminated for clarity and the 

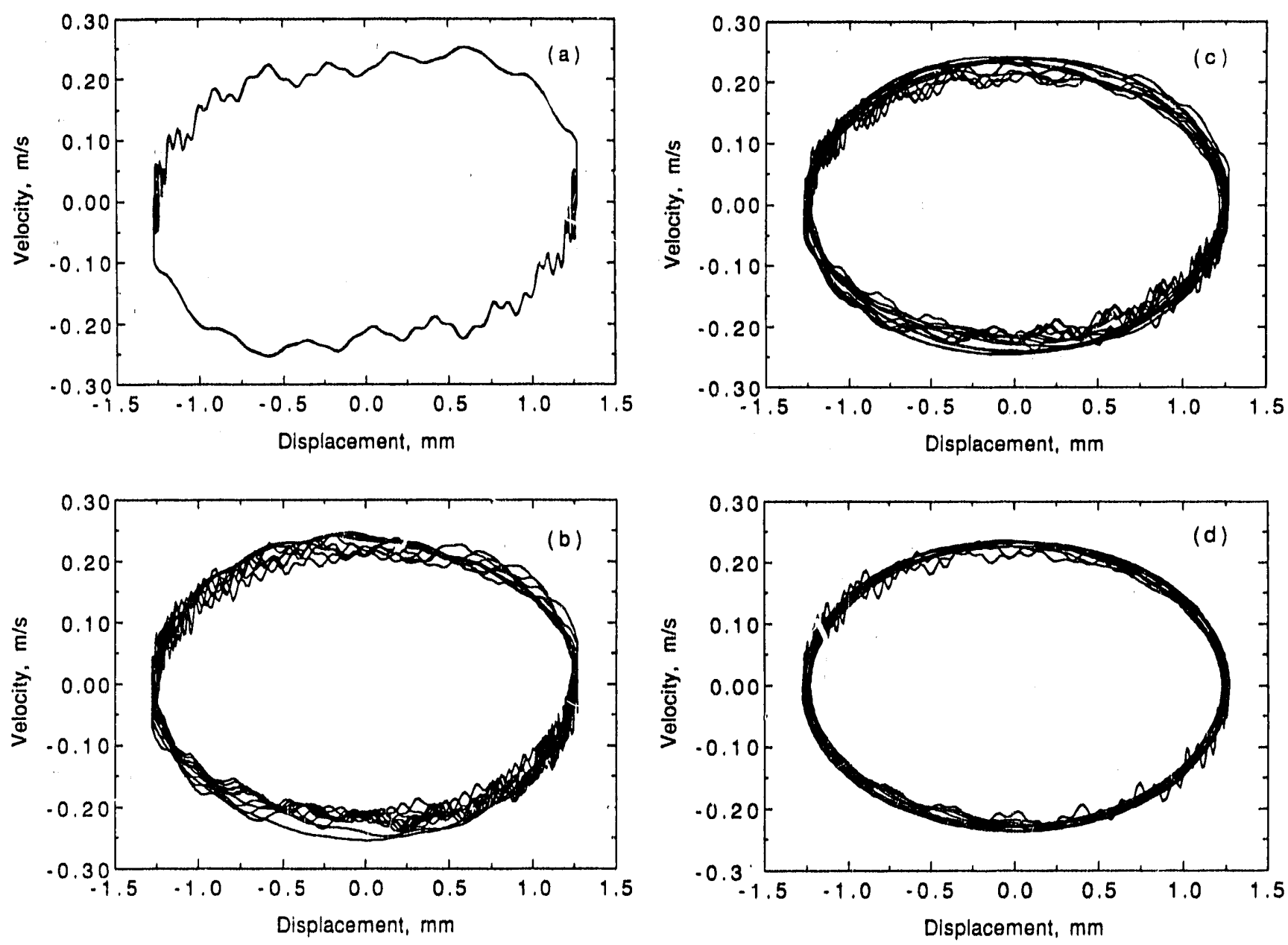

Fig. 2. Phase flow portraits at $\xi=1.0$ with (a) $U_{m l}=2.1 \mathrm{~m} / \mathrm{s}$, (b) $U_{m}=2.0 \mathrm{~m} / \mathrm{s}$, (c) $U_{m}=1.85 \mathrm{~m} / \mathrm{s}$, and (d) $U_{m}=1.80 \mathrm{~m} / \mathrm{s}$

diagrams show only the dynamics of the "steady state.' Because the span of the tube support is asymmetric (see Fig. 1) and the system is considered a multipledegree-of-freedom system, 10 modes were utilized to obtrin accurate simulations. In Fig. 2a, the phase portrait at $\xi=1.0$ for $U_{m}=2.1 \mathrm{~m} / \mathrm{s}$ is not an ellipse (as is that calculated by a single-degree-of-freedom system), but a complicated closed circle. At this flow velocity, the tube strikes the TSP twice in each circle, corresponding to periodic oscillations that repeat very well. As flow velocity decreased and the absolute value of negative damping decreased, the number of tube strikes was reduced and the strike period appeared to be irregular, at which time the phase portraits (see Figs. 2b, 2c, and $2 \mathrm{~d}$, for flow velocities $U_{m}=2.0,1.85$, and $1.80 \mathrm{~m} / \mathrm{s}$, respectively) appeared chaotic, i.e., in a kind of limited-band chaos. These chaotic motions apparently depend on the number of strikes during certain cycles, while strikes depend on the flow velocity, initial conditions, and other parameters, such as clearance and contact stiffness. Apparently, the number of strikes during certain cycles is unpredictable in this region. 


\subsection{Power Spectral Density}

Figure 3 shows power spectra of tube motions at different flow velocities. Because the fundamental oscillation frequencies within this flow velocity region are approximately the same, i.e., $f_{1}=29.6 \mathrm{~Hz}$ for the system shown in Figure 1, a nondimensional frequency $\mathrm{f} / \mathrm{f}_{1}$ was adopted in Fig. 3. Figure 3a shows the power spectral density (PSD) of the displacement at $U_{m}=2.1 \mathrm{~m} / \mathrm{s}$, which appears to be periodic with super harmonic components, $f / f_{1}=1,3,5, \ldots$ This is caused by regular striking of the tube. Figures $3 b-3 d$ for flow velocities $U_{m}=2.0,1.85$, and $1.80 \mathrm{~m} / \mathrm{s}$ present characteristics of limited-band chaos; the power spectra show a continuous process, although the fundamental frequency and superharmonic frequencies are rtill discernible.
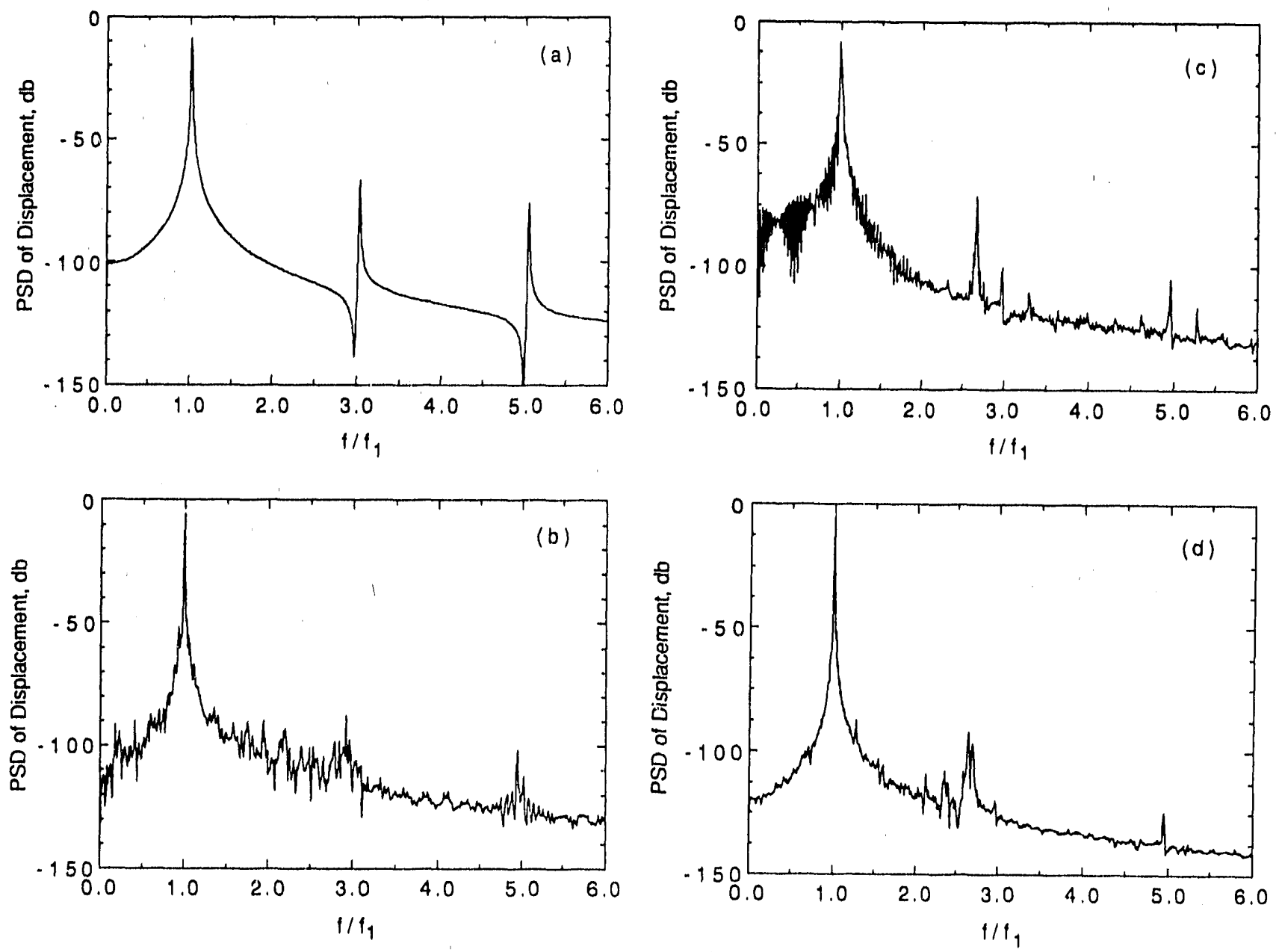

Fig. 3. Power spectral density of tube displacement with (a) $U_{m}=2.1 \mathrm{~m} / \mathrm{s}$, (b) $U_{m}=2.0 \mathrm{~m} / \mathrm{s}$, (c) $U_{m}=1.85 \mathrm{~m} / \mathrm{s}$, and (d) $U_{m}=1.80 \mathrm{~m} / \mathrm{s}$ 


\subsection{Poincaré Map}

The use of a Poincaré map is a powerful technique for distinguishing chaotic responses from periodic responses. The map represents a discretization of a continuous flow in the phase space of a dynamic system. A more general concept of the Poincaré map, valid for autonomous systems, has been given by Dowell (1984). The strobed points in the Poincaré map give an immediate answer about the periodicity of the response. The Poincaré map of a harmonic motion is a single point in the phase plane. A subharmonic motion of order $\mathbf{N}$ has a Poincaré map comprising $\mathrm{N}$ points (its period is $\mathrm{N}$ times the period of excitation). While in the case of chaos, the map has a complex fractal structure (Moon, 1987).

For an autonomous system, one must choose some plane in the phase space, transverse to the flow, and then obtain a Poincaré section (Moon, 1987). Using the same technique to choose a plane as done in our previous study (Cai and Chen, 1991), we chose the sampling trigger signal, say when $\dot{u}(1.0, t)=0$ and $u(1.0, t)<0$, shown in Fig. 4, at which point the values of $U(0.371, t)$ and $\dot{u}(0.371, t)$ would be saved (here $\xi=0.371$ means the midpoint in the first span of the tube between $\mathrm{C} 1$ and C2). Figures 5a-5d show such Poincaré maps, corresponding to flow velocities $U_{m}=2.1,2.0,1.85$, and $1.80 \mathrm{~m} / \mathrm{s}$, respectively. Figure $5 \mathrm{a}$ presents periodic motion because there is only a single point in the phase plane. However, Figs. $5 \mathrm{~b}$ to $5 \mathrm{~d}$ show limited-band chaotic motion with points scattered over a wide range. In these cases, although the Poincaré sections do not display artistic merit because there are a limited number of data points, they nevertheless seem to have a certain structure and confirm the existence of chaotic motion in this flow velocity range.

Another way to generate the Poincare maps in this study is to imagine the tube motion as a trajectory in a three-dimensional phase space (Fig. 6). Thus, if $u$ and $\dot{u}$ are the displacement and velocity of tube motion, $\left(u, \dot{u}, \omega_{1} t\right)$ represents a point in a cylindrical phase space where $\omega_{1}$ is fundamental frequency and $\phi=\omega_{1} t$ represents the phase of tube motion. A Poincaré map consists of sampled points in this three-dimensional space, for example, $\left[u\left(\xi, t_{n}\right), \dot{u}\left(\xi, t_{n}\right), \omega t_{n}=\phi_{0}+2 \pi n\right]$. Therefore, this map can be thought of as a slice through a torus (see Fig. 6).

As noted by Moon (1987), chaotic phase plane trajectories can often be unraveled with the Poincaré map by taking a set of pictures for different phase $\phi_{0}$. This is tantamount to sweeping the Poincaré plane in Fig. 6. While one Poincaré map can be used to expose the fractal nature of the attractor, a complete set of maps varying $\phi_{0}$ from 0 to $2 \pi$ is sometimes needed to obtain a complete picture of the attractor on which the motion is riding. 

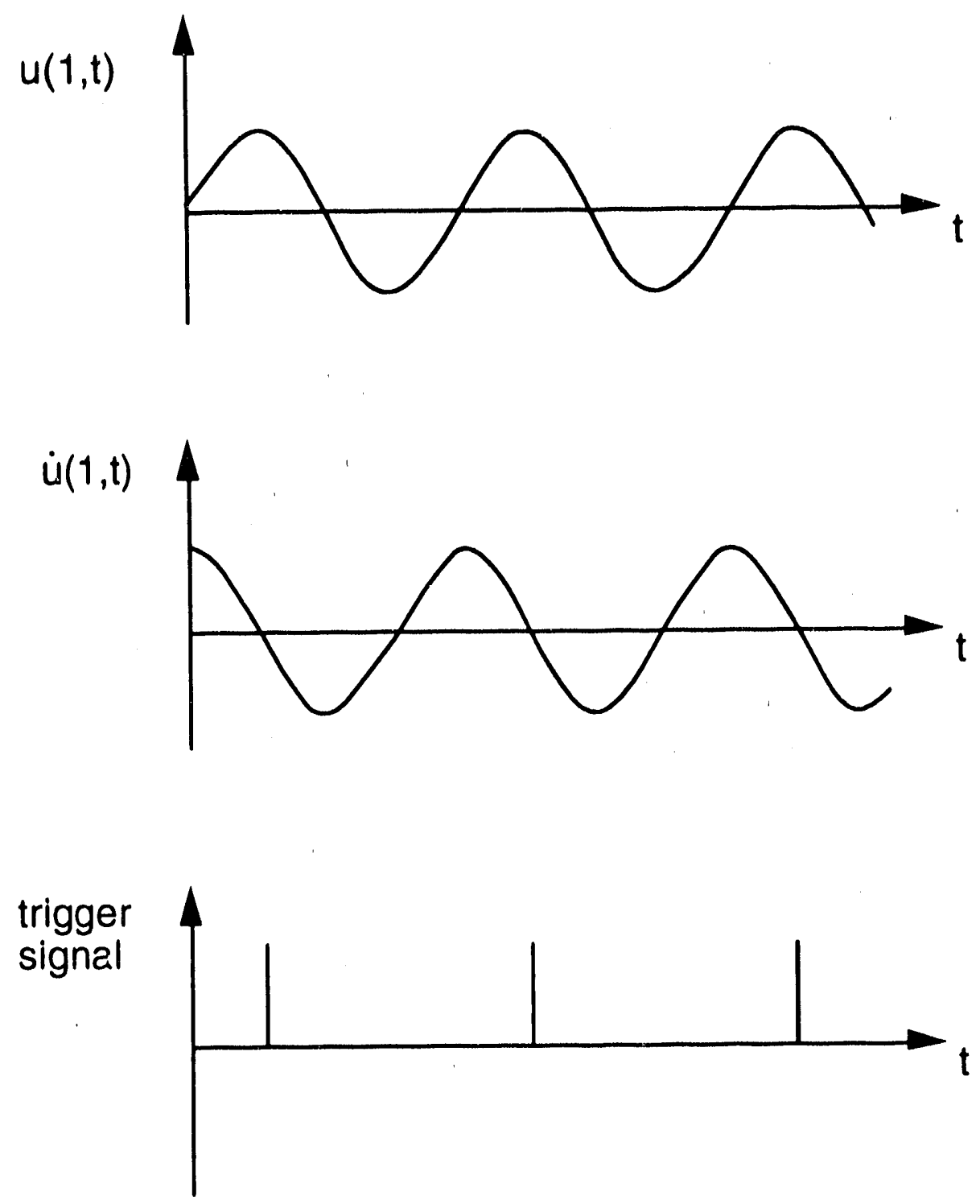

Fig. 4. Sampling trigger signal for Poincaré map

With flow velocity $U_{m}=1.80 \mathrm{~m} / \mathrm{s}$, at which tube motion has been predicted as chaotic (see Fig. 5d), we chose $\phi=0$ at $t=t_{0}$ when the transient has died out and $\dot{\mathrm{u}}\left(1.0, \mathrm{t}_{0}\right)=0$. Then a series of pictures (Fig. 7) is produced of various cross sections of a chaotic torus motion in the three-dimensional phase space.

\subsection{Lyapunov Exponent}

Lyapunov exponents have been shown to be the most useful dynamic diagnostic tool in determining chaotic systems quantitatively. Lyapunov exponents are the average exponential rates of divergence or convergence of 

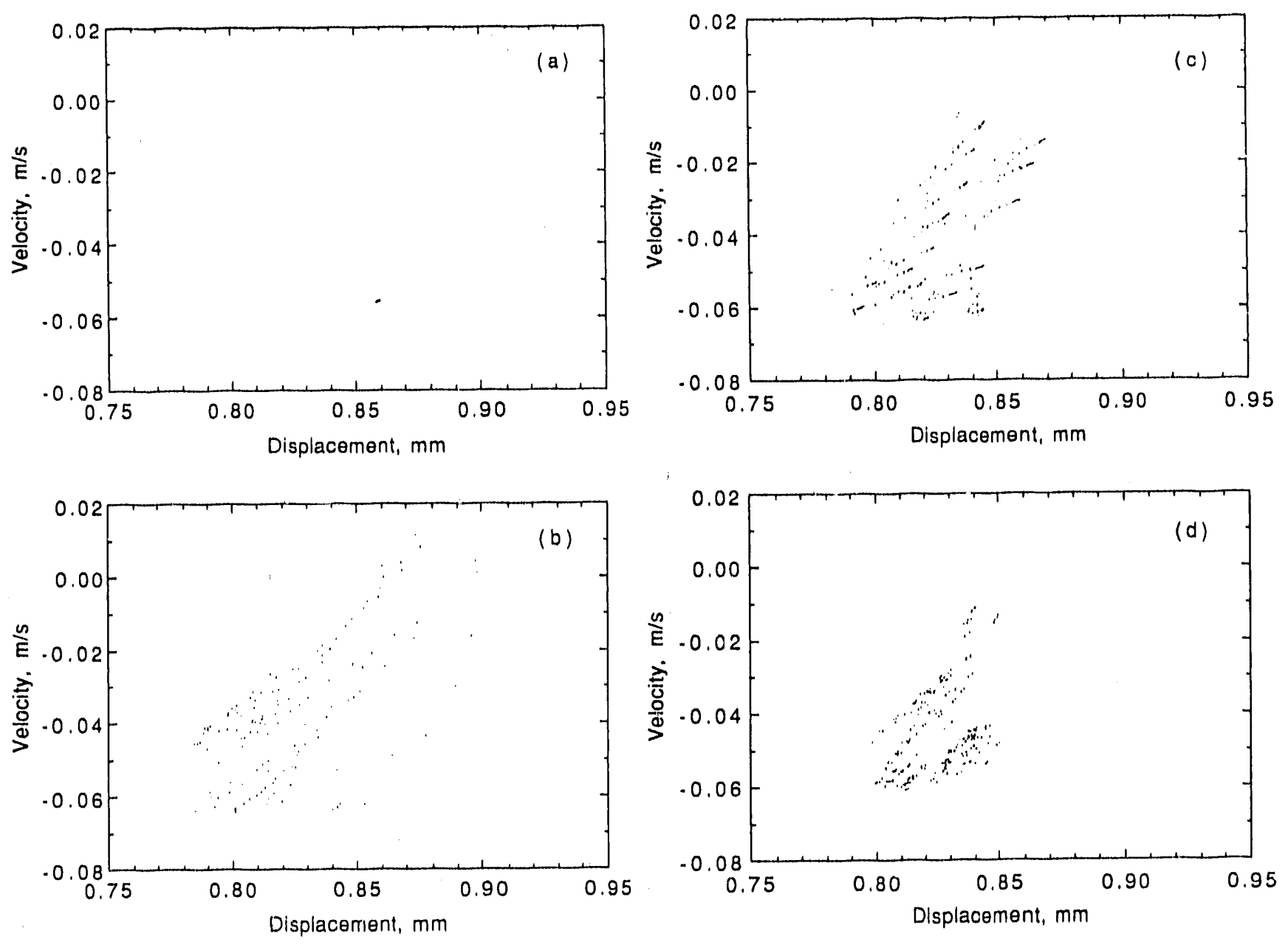

Fig. 5. Poincaré map of tube motion at $\xi=0.371$ with (a) $U_{m}=2.1 \mathrm{~m} / \mathrm{s}$,

(b) $U_{m}=2.0 \mathrm{~m} / \mathrm{s}$, (c) $U_{m}=1.85 \mathrm{~m} / \mathrm{s}$, and (d) $U_{m}=1.80 \mathrm{~m} / \mathrm{s}$

nearby orbits in phase space. This technique gives a quantitative measure of the corresponding motions: for example, the motion is periodic if the exponent is negative or zero, but chaotic if it is positive. Any system containing at least one positive Lyapunov exponent is defined as chaotic. We use the algorithms proposed by Wolf et al. (1985) to determine the Lyapunov exponents from a time series of tube motion. Figure 8 shows the results of Lyapunov exponents at different flow velocities. In Fig. $8 \mathrm{a}$, with $\mathrm{U}_{\mathrm{m}}=2.1 \mathrm{~m} / \mathrm{s}$, Lyapunov exponents are regative, corresponding to periodic motion of the tube. In Figs. $8 b-8 d$, with $U_{m}=2.0,1.85$, and $1.80 \mathrm{~m} / \mathrm{s}$, respectively, Lyapunov exponents are positive, constituting convincing evidence for the existence of chaotic motion in this instability region of the TSP-inactive mode. 


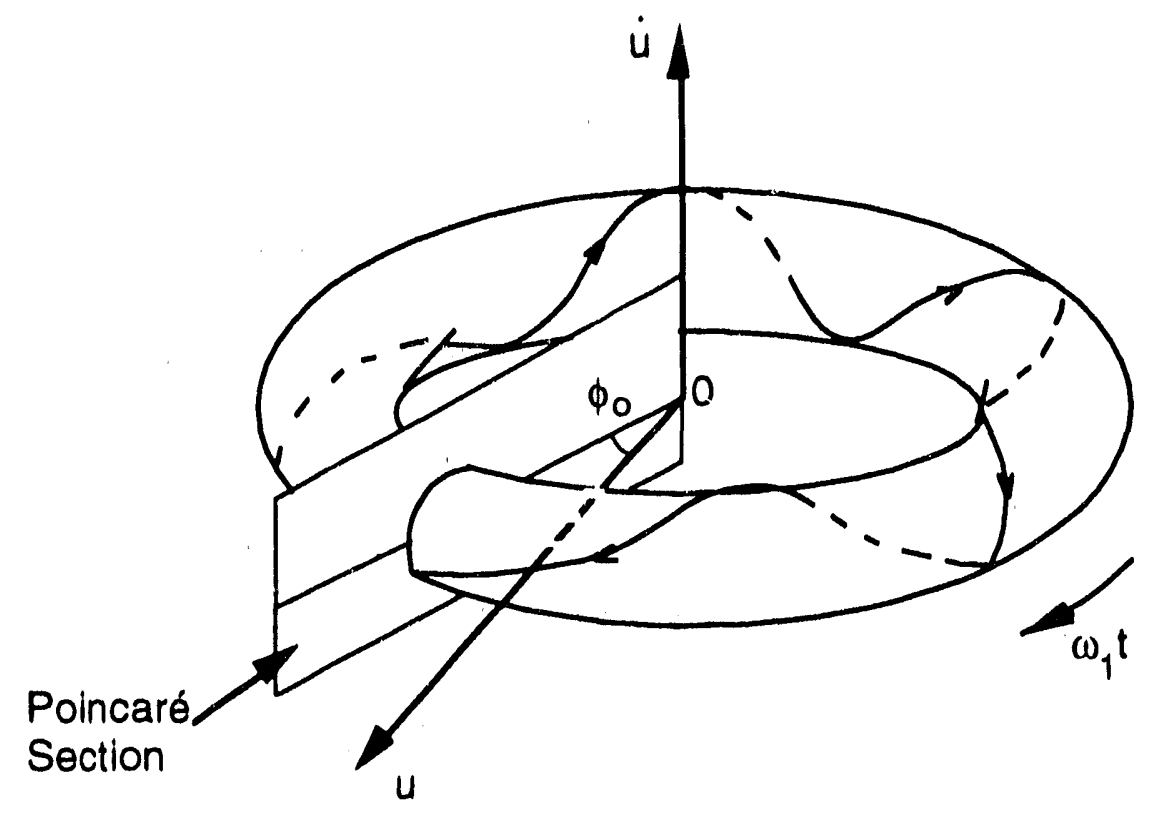

Fig. 6. Geometric interpretation of Poincare sections in the three-dimensional phase space

\subsection{Fractal Dimensions}

Another approach for predicting chaotic motion quantitatively is the use of fractal dimensions. A noninteger fractal dimension of the orbit in a phase space implies the existence of a strange attractor. The basic idea is to characterize the "strangeness" of the chaotic attractor. While practical use of the fractal dimensions in measuring and characterizing chaotic motion has yet to be fully established, various definitions have been developed, including the capacity dimension, correlation dimension, and information dimension (Baker and Gollub, 1990). In many cases, it is sufficient to estabiish that the dimension is not, an integer or that the attractor is indeed strange. To our knowledge, this is the first application of the attractor dimension to fluidelastic instability of a loosely supported tube in crossflow.

Of the several methods available to estimate the attractor dimensions, we used measurement of the correlation dimension, which has been used successfully by many investigators in other fields (Moon, 1985). Grassberger and Proccacia (1983) studied this definition of dimension extensively. In this method, a correlation function $\mathrm{C}(\mathrm{r})$ can be calculated by constructing a sphere or length $r$ at each point $x_{i}$ in phase space and counting the number of points in each sphere, that is, 

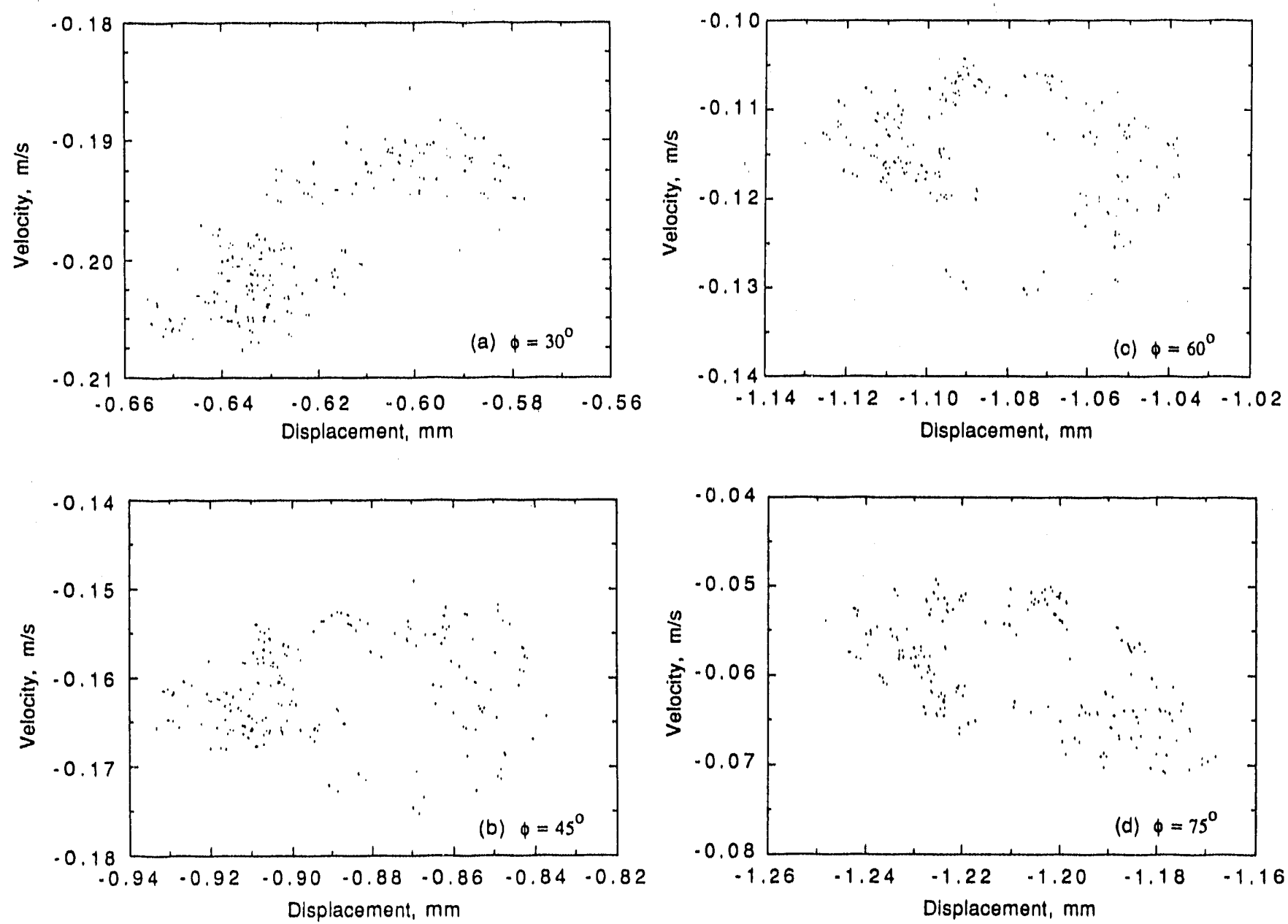

Fig. 7. Poincaré map of tube motion at $\xi=1.0$ with $U_{m}=1.80 \mathrm{~m} / \mathrm{s}$ for various phases (a) $\phi=30$, , (b) $\phi=45^{\circ}$, (c) $\phi=60^{\circ}$, (d) $\phi=75^{\circ}$, (e) $\phi=-30^{\circ}$, (f) $\phi=-45^{\circ}$, (g) $\phi=-60^{\circ}$, and (h) $\phi=-75^{\circ}$

$$
C(r)=\lim _{N \rightarrow \infty} \frac{1}{N_{0}^{2}} \sum_{i}^{N} \sum_{j}^{N} H\left(r-\left|x_{i}-x_{j}\right|\right),
$$

where $H(s)=1$ if $s>0$ and $H(s)=0$ if $s<0$. For many attractors, this function has been found to exhibit a power law dependence on $r$ as $r \rightarrow 0$, that is

$$
\lim _{r \rightarrow 0} C(r)=a r^{d}
$$

so that we may define a fractal or correlation dimension with the slope of the $\log \mathrm{C}$ versus $\log r$ curve 

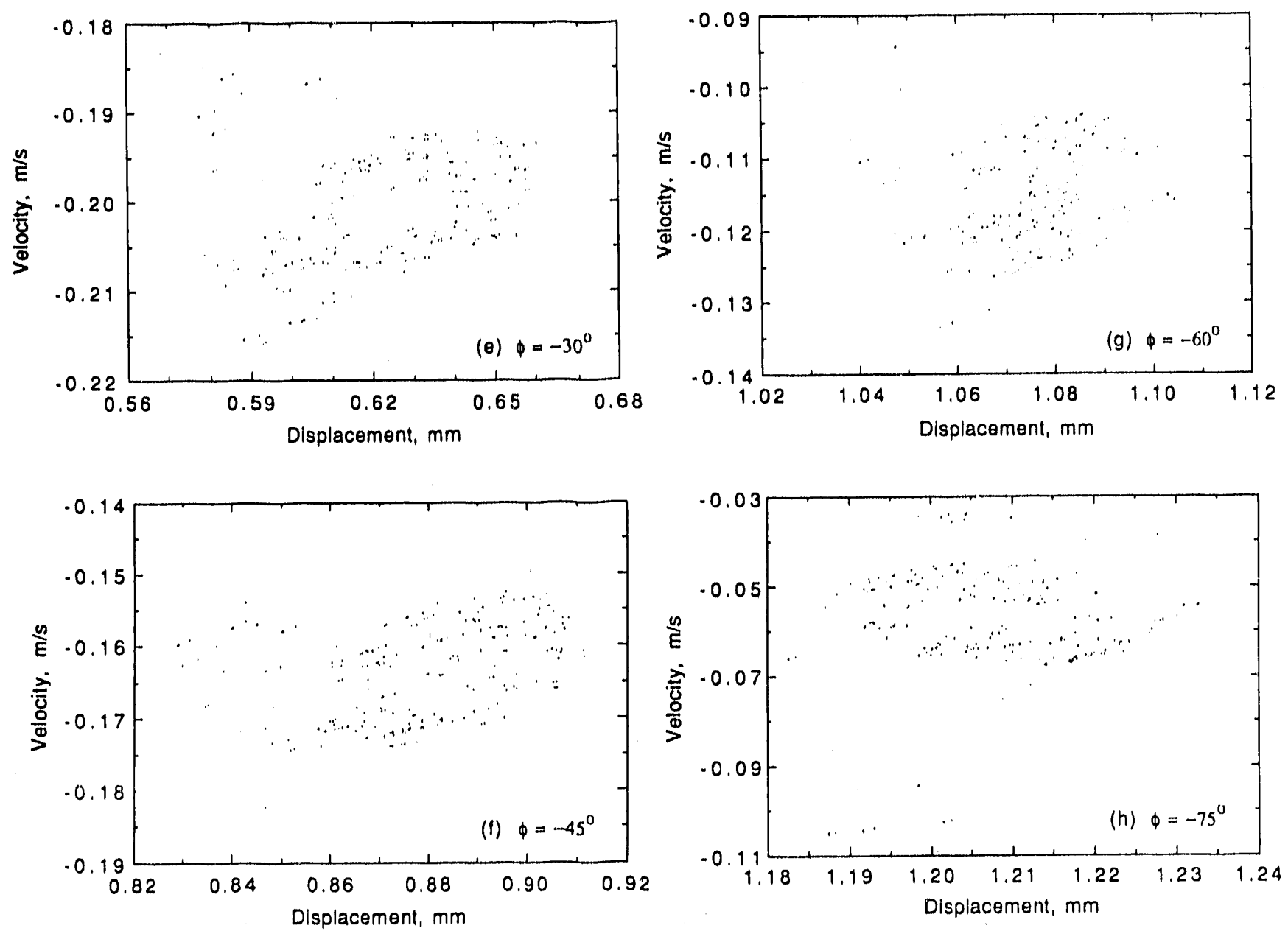

Fig. 7. (Cont'd)

$$
\mathrm{d}_{\mathrm{G}}=\lim _{\mathrm{r} \rightarrow 0} \frac{\log \mathrm{C}(\mathrm{r})}{\log \mathrm{r}}
$$

Using this technique, we can compute the correlation dimension of chaotic motion of the tube from the data of the Poincaré map in Fig. $5 \mathrm{~b}$ (with flow velocity $\left.\mathrm{U}_{\mathrm{m}}=2.0 \mathrm{~m} / \mathrm{s}\right)$. Figure 9 shows the logarithm of the correlation function $\mathrm{C}(\mathrm{r})$ versus $\log r$, and Fig. 10 shows the local slope of $\mathrm{C}(\mathbf{r})$. The slope for the intermediate values of $r$ is $\approx 1.55$. This fractal dimension is convincing evidence that the attractor is indeed strange when the tube is vibrating in this flow velocity range.

Many investigators have suggested another definition of fractal dimension, (i.e., the information dimension) that is similar to the capacity dimension but attempts to account for the frequency with which the trajectory visits each 

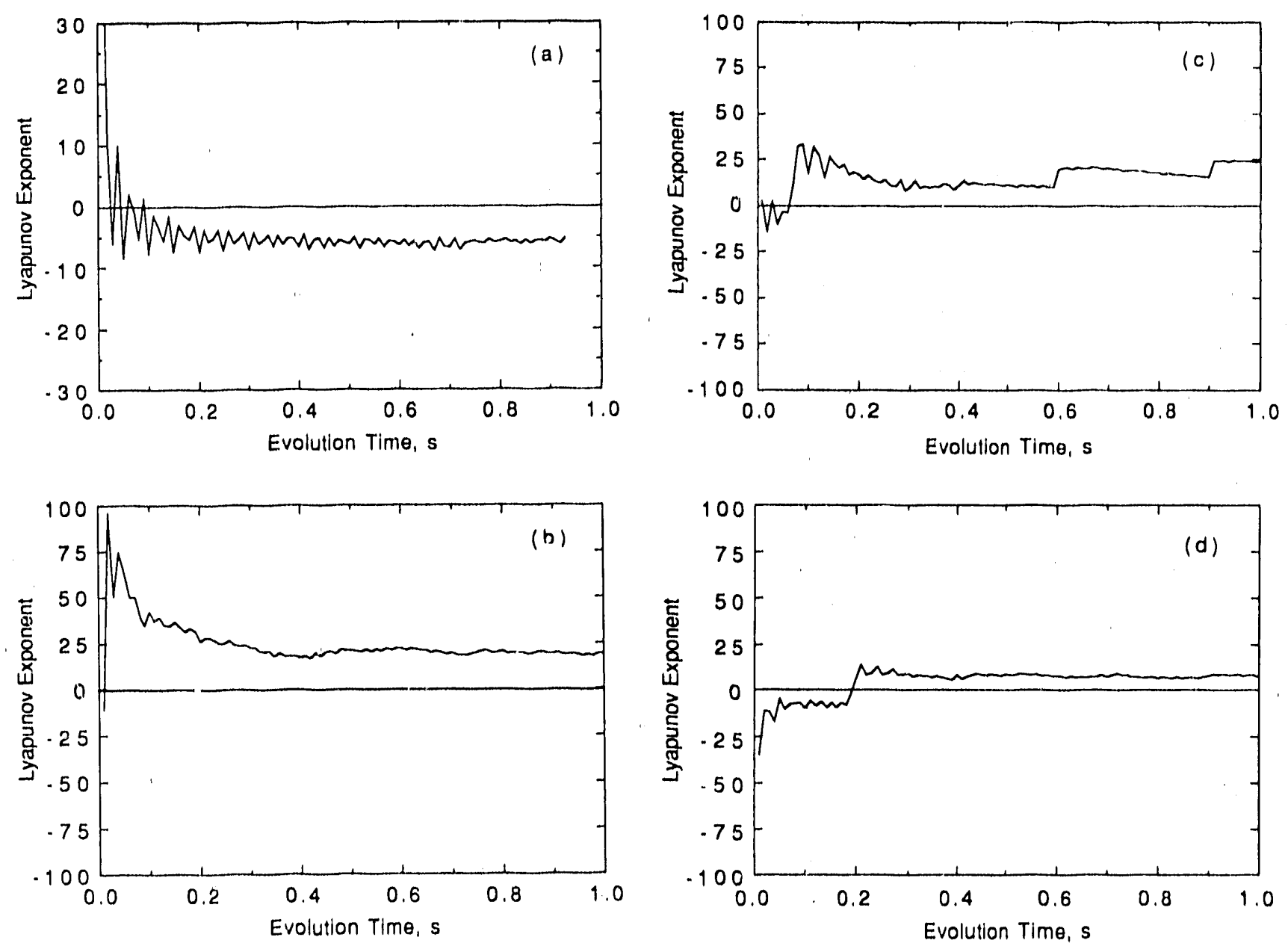

Fig. 8. Lyapunov exponents of tube motion with (a) $U_{m}=2.1 \mathrm{~m} / \mathrm{s}$, (b) $U_{m}=$ $2.0 \mathrm{~m} / \mathrm{s}$, (c) $U_{m}=1.85 \mathrm{~m} / \mathrm{s}$, and $(d) U_{m}=1.80 \mathrm{~m} / \mathrm{s}$. (Embedding dimension is selected to be 6 and delay time to be $1 / 2$ of the mean orbital period.)

covering cube. To calculate the information dimension, we count the number of points $N_{i}$ in each of the $N$ cells and the probability of finding a point in that cell $P_{i}$, where

$$
P_{i} \equiv \frac{N_{i}}{N_{0}}, \quad \sum^{N} P_{i}=1
$$

where $N_{0}$ is the total number of points in the set. Note that $N_{0} \neq N$. The information entropy is defined by the expression

$$
I(r)=-\sum_{i=1}^{N} P_{i} \log P_{i}
$$




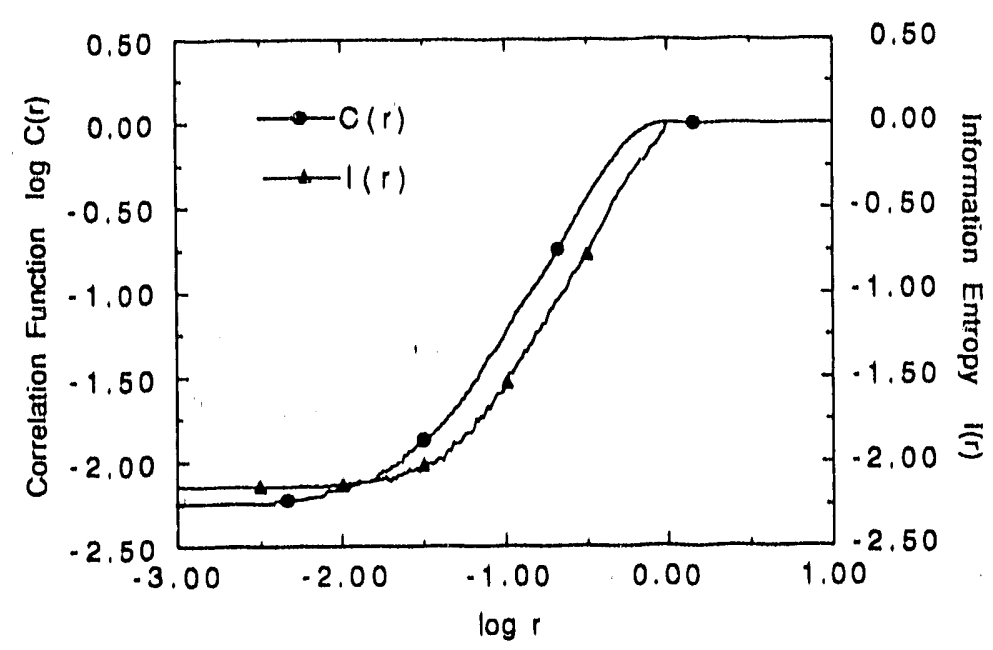

Fig. 9. Correlation function and information entropy for set of points in Poincaré map in Fig. $5 b$

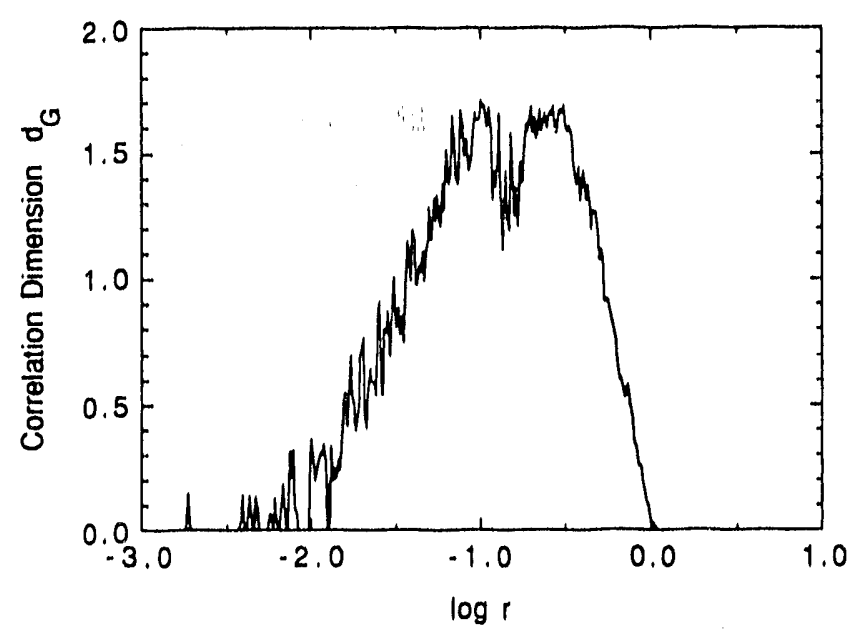

Fig. 10. Correlation dimension: local slope of correlation function in Fig. 9

For small $r$, it is found that $I$ behaves as

$$
\mathrm{I} \approx \mathrm{d}_{\mathrm{I}} \log (1 / \mathrm{r})
$$

so that for small $r$ we may define a dimension

$$
d_{I}=\lim _{r \rightarrow 0} \frac{I(r)}{\log (1 / r)}=\lim _{r \rightarrow 0} \frac{\sum P_{i} \log P_{i}}{\log r} .
$$


We calculated the information entropy $I(r)$ from the Poincaré section of Fig, $5 b$ (with flow velocity $U_{m}=2.0 \mathrm{~m} / \mathrm{s}$ ). The result of $I(r)$ is shown in Fig. 9 and is compared with the correlation function $\log \mathrm{C}(\mathrm{r})$. From the slopes of the information entropy $I(r)$ and the correlation function $C(r)$, we find that the information dimension and the correlation dimension are very close. These results again display the existence of the strange attractor in the system.

\subsection{Bifurcation Diagram}

A widely used technique for examining the pre- or postchaotic changes in a dynamic system under parameter variations is the bifurcation diagram, from which we may find a route to chaos, namely a route from periodic to chaotic motions through parameter changes. With fast computers available, it is easy and helpful to vary the control paranieters to obtain a bifurcation diagram. From this diagram, we can see if the system displays steady, periodic, or chaotic behavior for some continuous range of flow velocity to obtain a full understanding of the system dynamics. In this way, we can have confidence in deciding more definitely whether the system becomes chaotic. Also, we can observe and pinpoint sudden changes in system behavior. Both the control parameter and the output signal must be carefully chosen to ensure that they can provide sufficient information.

In our case, flow velocity was chosen as the control parameter and tube displacement as the output signal. The difficulty was in determining the locations of the triggering and output signals to produce an easily interpreted bifurcation diagram. After many results were examined, the velocity of tube moticin at location $\mathrm{C} 3(\xi=1.0)$ was taken as the triggering signal and the displacement at location $\xi=1.0$ was taken as the output signal. When the triggeing signal was equal to zero, $\dot{\mathrm{u}}(1.0, \mathrm{t})=0$, and tube displacement at $\xi=1.0$ was negative, $\mathrm{u}(1,0, \mathrm{t})<0$, the values of $\mathrm{u}(1,0, \mathrm{t})$ were recorded. By slow variation of flow velocity, the bifurcation diagram shown in Fig. 11 was produced. In Fig. 11, a symmetric clearance $\mathrm{e}_{1}=\mathrm{e}_{2}=1.27 \mathrm{~mm}$ at the TSP was applied.

It is clear in Fig. 11 that for flow velocity less than the first critical flow velociby $U_{m}=1.77 \mathrm{~m} / \mathrm{s}$ or higher than the second critical flow velocity $U_{m}=$ $4.73 \mathrm{~m} / \mathrm{s}$ (for details, see Cai and Chen, 1991), all oscillations died out as time increased; when flow velocity reached the critical values, there was a jump in displacement (i.e., the Hopf bifurcation occurred). When the tube lost its stability and struck the TSP, chaotic motion occurred. But as flow velocity reached certain values between 2.02 and $4.17 \mathrm{~m} / \mathrm{s}$, the tube struck the TSP regularly in a harmonic periodic vibration because of the damping-controlled instability of the tube. Therefore, tube motion with the above system parameters can be described as follows: first a region of chaotic motion $\left(U_{m}=1.77\right.$ to $2.02 \mathrm{~m} / \mathrm{s}$ ), a region of periodic 


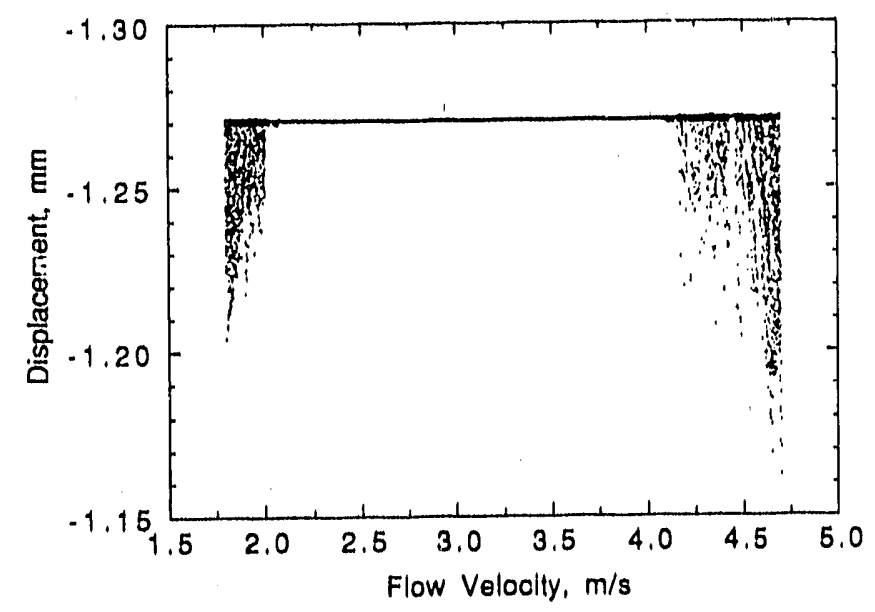

Fig. 11. Bifurcation diagram of tube motion in instability region

motion $\left(U_{m}=2.02\right.$ to $\left.4.17 \mathrm{~m} / \mathrm{s}\right)$, and a second region of chaotic motion $\left(U_{m}=4.17\right.$ to $4.77 \mathrm{~m} / \mathrm{s}$ ). This distribution of periodic/chaotic motion corresponds to negative system damping. According to Eq. 3, system damping depends on flow velocity and fluid force coefficients, namely

$$
\zeta_{i}=\left[\left(2 \zeta_{n} \omega_{i n}-\frac{\gamma U_{r}^{2}}{\pi^{3}} \omega_{i} \alpha_{\text {in }}^{d}\right) /\left(2 \omega_{i}\right)\right]
$$

or

$$
\begin{aligned}
& \zeta_{i}=\left[\left(2 \zeta_{n} \omega_{\text {in }}-\frac{\gamma U_{m}^{2}}{\pi R^{3} \omega_{i}} \alpha_{i n}^{d}\right) /\left(2 \omega_{i}\right)\right] \\
& i=1,2,
\end{aligned}
$$

where fluid force coefficients are also functions of flow velocity and $\omega_{i}$ (Eq. 6). When the flow velocity is in a certain range, system damping may become negative and tube motion becomes unstable. System dampings as functions of mean flow velocity for Models 1 and 2 are shown in Figs, 12a and 12b, respectively. We find that the critical mean flow velocities $\left(\zeta_{1}=0\right)$ for Model 1 are $U_{m}=1.77 \mathrm{~m} / \mathrm{s}$ and $4.73 \mathrm{~m} / \mathrm{s}$, corresponding to fundamental frequencies $f_{1}=? 9.28 \mathrm{~Hz}$ and 33.85 $\mathrm{Hz}$ (Fig. 12a); and the critical mean flow velocities $\left(\zeta_{2}=0\right)$ for Model 2 are $U_{m}=$ $2.31 \mathrm{~m} / \mathrm{s}$ and $7.41 \mathrm{~m} / \mathrm{s}$, corresponding to fundamental frequencies $\mathrm{f}_{2}=41.58 \mathrm{~Hz}$ and $52.03 \mathrm{~Hz}$. Note that the fundamental frequencies are iterated from Eq. 6 and are different from the natural frequencies $\left(f_{\text {in }}=\omega_{\text {in }} / 2 \pi\right.$, for example, in our case $\mathrm{f}_{11}=32.73 \mathrm{~Hz}, \mathrm{f}_{21}=48.70 \mathrm{~Hz}$ ). In our case, Model 1 dominates the tube motion. Thus, instability of tube motion will depend on the system damping of Model 1, so 

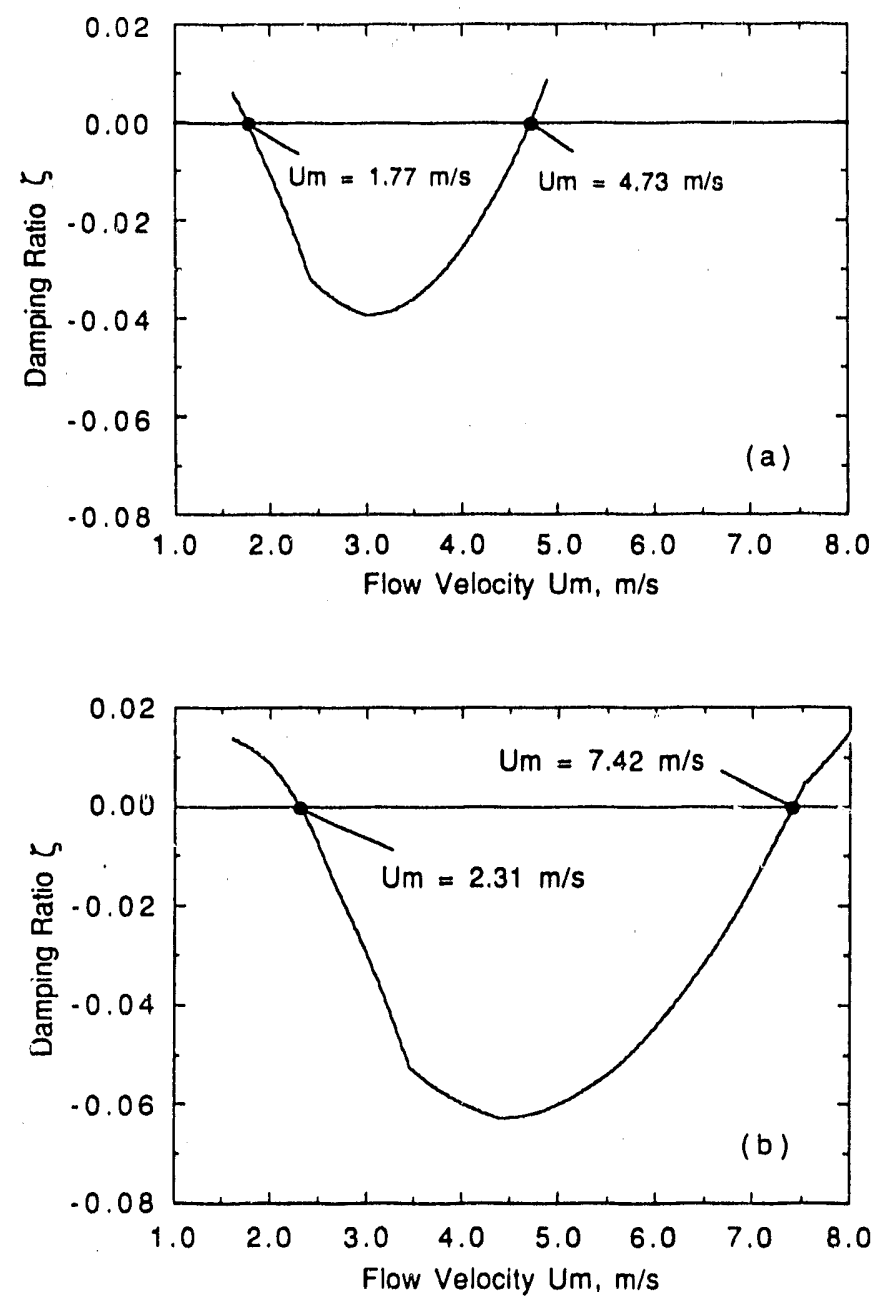

Fig. 12. System damping ratio vs. mean flow velocity (a) Model 1 and (b) Model 2

that we find good agreement between the bifurcation diagram and system damping when comparing Fig. 11 with Fig. 12a. When the absolute negative damping is below certain values, tube motion is chaotic, but when it exceeds those values it develops into periodic harmonic vibrations.

It is quite interesting that the second chaotic region seems to be wider than the first and that there are windows in the second chaotic region. So far, we find it difficult to interpret these phenomena. Figures 13 and 14 show PSDs, Poincaré maps, and Lyapunov exponents with flow velocities $U_{m}=4.6 \mathrm{~m} / \mathrm{s}$ and $4.7 \mathrm{~m} / \mathrm{s}$, respectively, in the second chaotic region. Apparently, Fig. 14 indicates a chaotic motion for $U_{m}=4.7 \mathrm{~m} / \mathrm{s}$. However, Fig. 13 appears to be a typical quasiperiodic case: (a) The PSD shows a period-4 motion, as described in the previous work (Cai and Chen, 1991), and the frequencies appearing in the spectrum of quasiperiodic motion can be a combination of two incommensurate frequencies, i.e., 
20
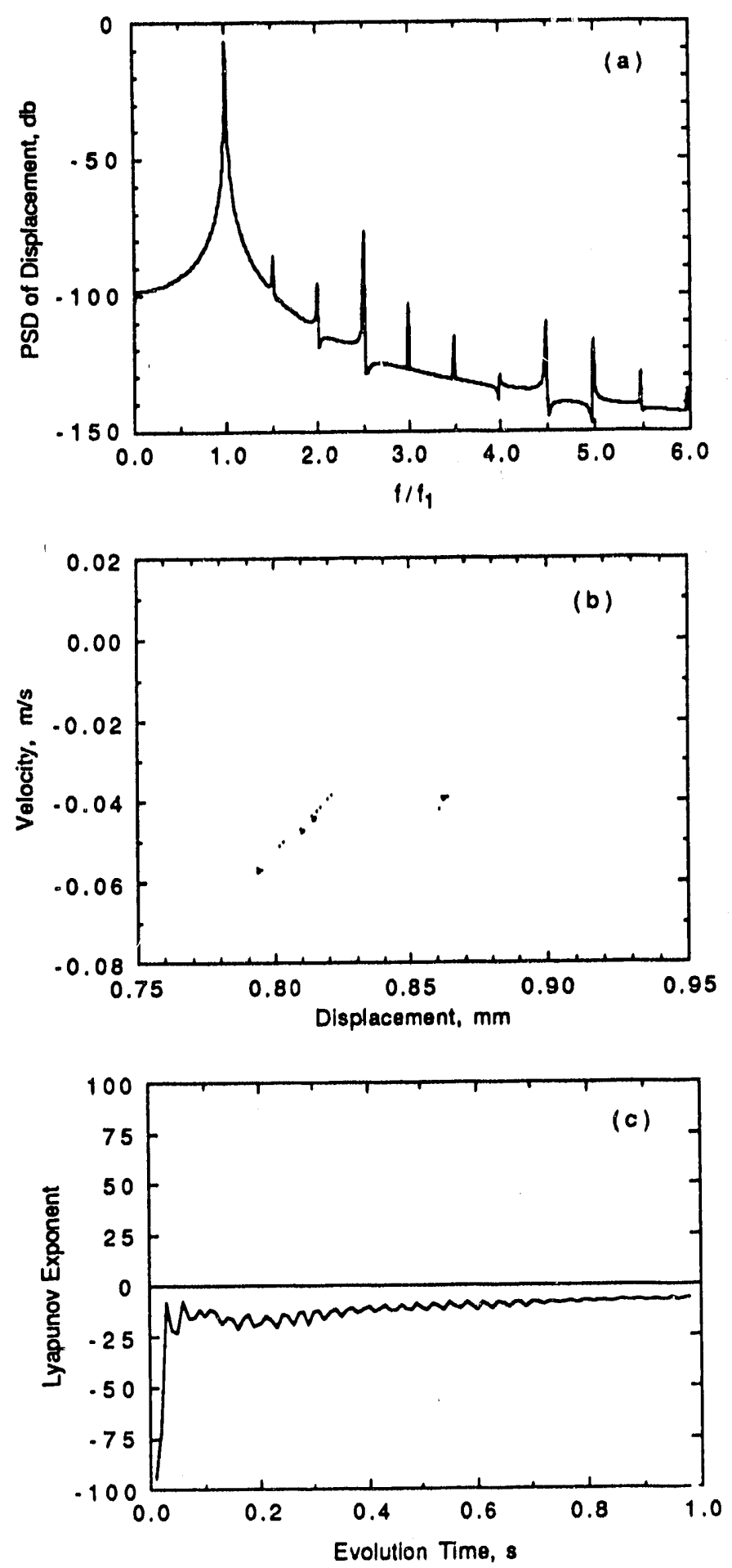

Fig. 13. PSD (a), Poincaré map (b), and Lyapunov exponent (c) of tube motion with $U_{m}=4.6 \mathrm{~m} / \mathrm{s}$ 
21
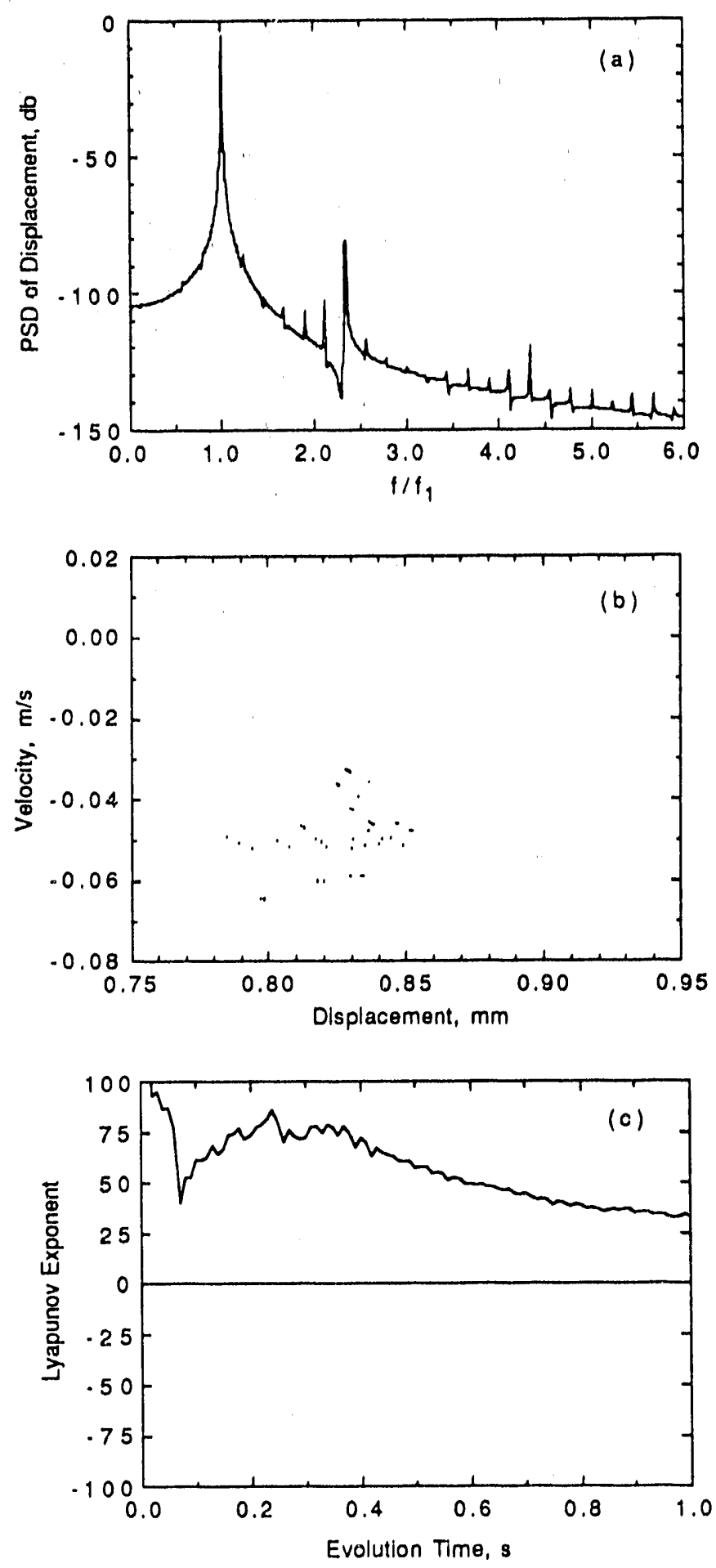

Fig. 14. PSD (a), Poincaré map (b), and Lyapunov exponent (c) of tube motion with $U_{m}=4.7 \mathrm{~m} / \mathrm{s}$ 


$$
f_{(m, n)}=m f_{(1)}+n f_{(2)}
$$

In Fig. 13a, $f_{(1)}=1.0 f_{1}$ and $f_{(2)}=1.5 f_{1}$. Therefore, peaks in Fig. 13a are $f_{(1,0)}, f_{(0,1)}$, $f_{(-1,2)}, f_{(4,-1)}, f_{(3,0)} \ldots$ (b) In Fig. $13 \mathrm{~b}$, four basins of attraction seem to exist in the Poincaré map. (c) The Lyapunov exponent in Fig. 13c is negative, indicating a subharmonic motion.

\subsection{Measurements with Asymmetric Gars at the TSP}

Many computations were carried out for various diametral clearances at the TSP, symmetric or asymmetric, because we recognized that the nonlinearity of the TSP plays a very important role in periodic and chaotic motions of the tube. Bifurcation diagrams in Fig. 15 give some results of these computations when asymmetric clearances were applied. In Fig. 15, we always set $e_{1}$ equal to 1.27 $\mathrm{mm}$, but e2 changed from 2.54 , to 1.45 , to 1.40 , and $1.35 \mathrm{~mm}$, corresponding to Figs. 15a, 15b, 15c and $15 \mathrm{~d}$, respectively. The flow velocity range and triggering and output signals were the same as those in Fig. 11. From Fig. 15, we noted that nonlinearity of those asymmetric gaps significantly affected the distribution of periodic and chaotic motions of the tube with various flow velocities in the instability region of the TSP-inactive mode. In Fig. 15a, the tube no longer struck the stop with the gap of $e_{2}$, because $e_{2}=2.54 \mathrm{~mm}$ is virtually an infinite gap under these system parameters. Thus, chaotic motion seems possible over almost the whole range when flow velocity varies. When $e_{2}=1.45 \mathrm{~mm}$ (Fig. 15b), the tube begins to strike the stop irregularly at the e 2 gap when flow velocity exceeds $U_{m}=$ $2.30 \mathrm{~m} / \mathrm{s}$. Hence, there is another chaotic motion in this case. When $\mathrm{e}_{2}=1.40 \mathrm{~mm}$ and $\mathrm{e}_{2}=1.35 \mathrm{~mm}$ (Figs. $15 \mathrm{c}$ and $15 \mathrm{~d}$ ), the tube begins to strike the stop regularly when flow velocities exceed some values and periodic and quasiperiodic motion is developing.

However, the situations in Fig. 15 are so complicated when asymmetric gaps are applied that we cannot predict the clear criteria from the chaotic, quasiperiodic-to-periodic motions by control parameters such as flow velocity, system damping, and clearance. Figures 16 and 17 give PSD of tube motion for $\mathrm{U}_{\mathrm{m}}=2.35$ and $2.80 \mathrm{~m} / \mathrm{s}$ with clearance corresponding to that in Fig. 15. Because the fundamental frequency and superharmonic frequencies are discernible in Figs. 16 and 17, the motion under these parameters appears to be quasiperiodic. Figure 16c $\left(\mathrm{U}_{\mathrm{m}}=2.35\right)$ and Fig. $17 \mathrm{c}\left(\mathrm{U}_{\mathrm{m}}=2.80 \mathrm{~m} / \mathrm{s}\right)$, with $\mathrm{e}_{1}=1.57 \mathrm{~mm}, \mathrm{e}_{2}=$ $1.40 \mathrm{~mm}$, appear to begin to develop chaotic motions because the spectra seem too close to a broad response. However, Figs. $17 \mathrm{~b}$ and $17 \mathrm{~d}$ are clearly of period-2 motion when $\mathrm{e}_{2}=1.45$ and $1.35 \mathrm{~mm}$ and $\mathrm{U}_{\mathrm{m}}=2.80 \mathrm{~m} / \mathrm{s}$ are applied. 

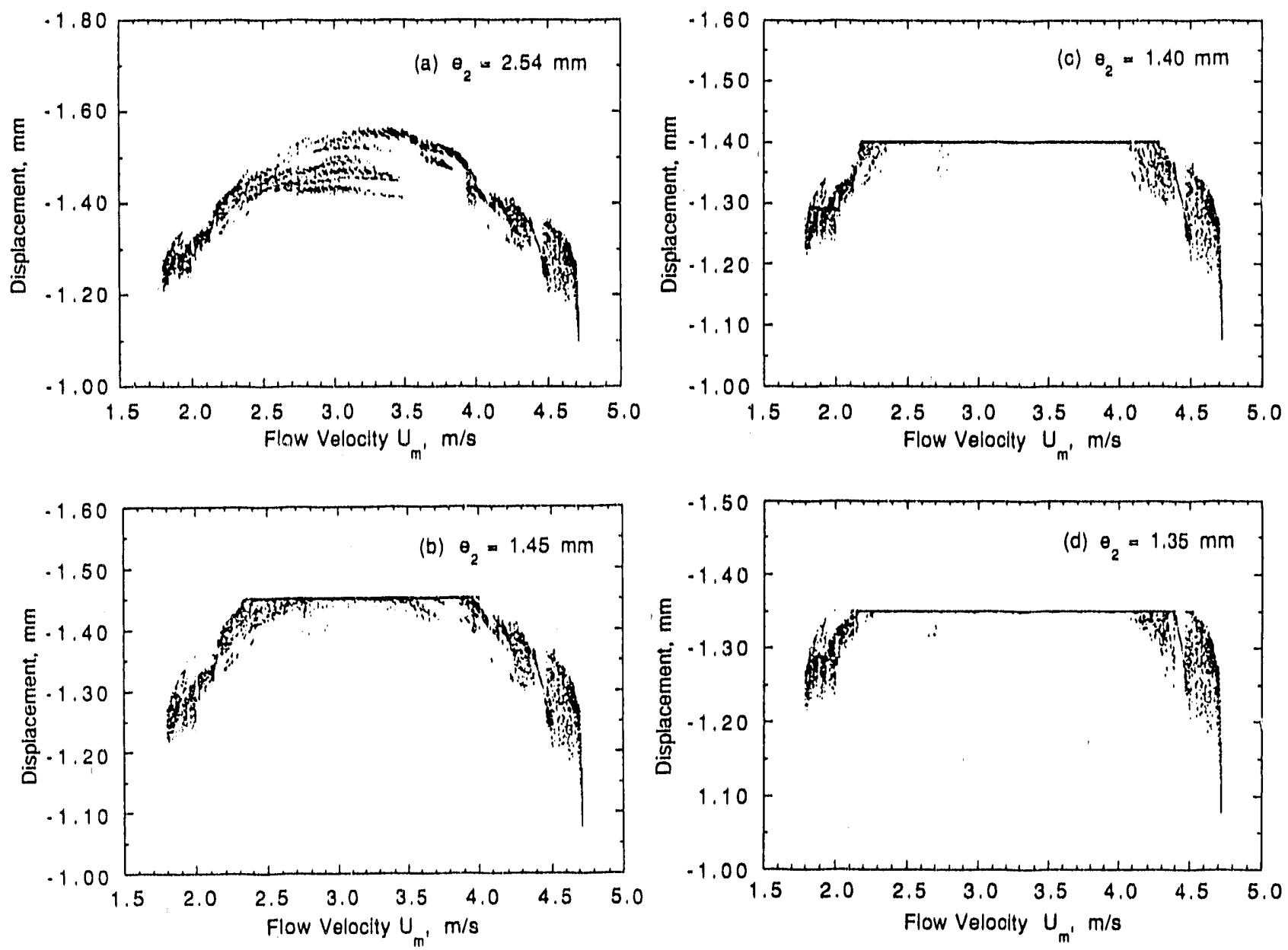

Fig. 15. Bifurcation diagrams of tube motion with asymmetric clearances $e_{1}=1.27 \mathrm{~mm}$ and $(a) e_{2}=2.54 \mathrm{~mm}$, (b) $e_{2}=1.45 \mathrm{~mm}$, (c) $e_{2}=1.40 \mathrm{~mm}$, and $(d) e_{2}=1.35 \mathrm{~mm}$

\section{Conclusions}

Based on a previously developed unsteady-flow theory and bilinear mathematical model for fluidelastic instability of loosely supported tubes subjected to nonuniform crossflow, an extensive analytical study was conducted of fluidelastic instability of tubes in the unstable region associated with the TSPinactive mode. Particular attention was given to the possible existence of chaotic oscillation. With typical nonlinear boundary conditions, i.e., symmetric or asymmetric gaps at the TSP, tube motion can be expressed by a nonlinear autonomous mechanical system in which chaotic motion is highly probable with variation of control parameters. 

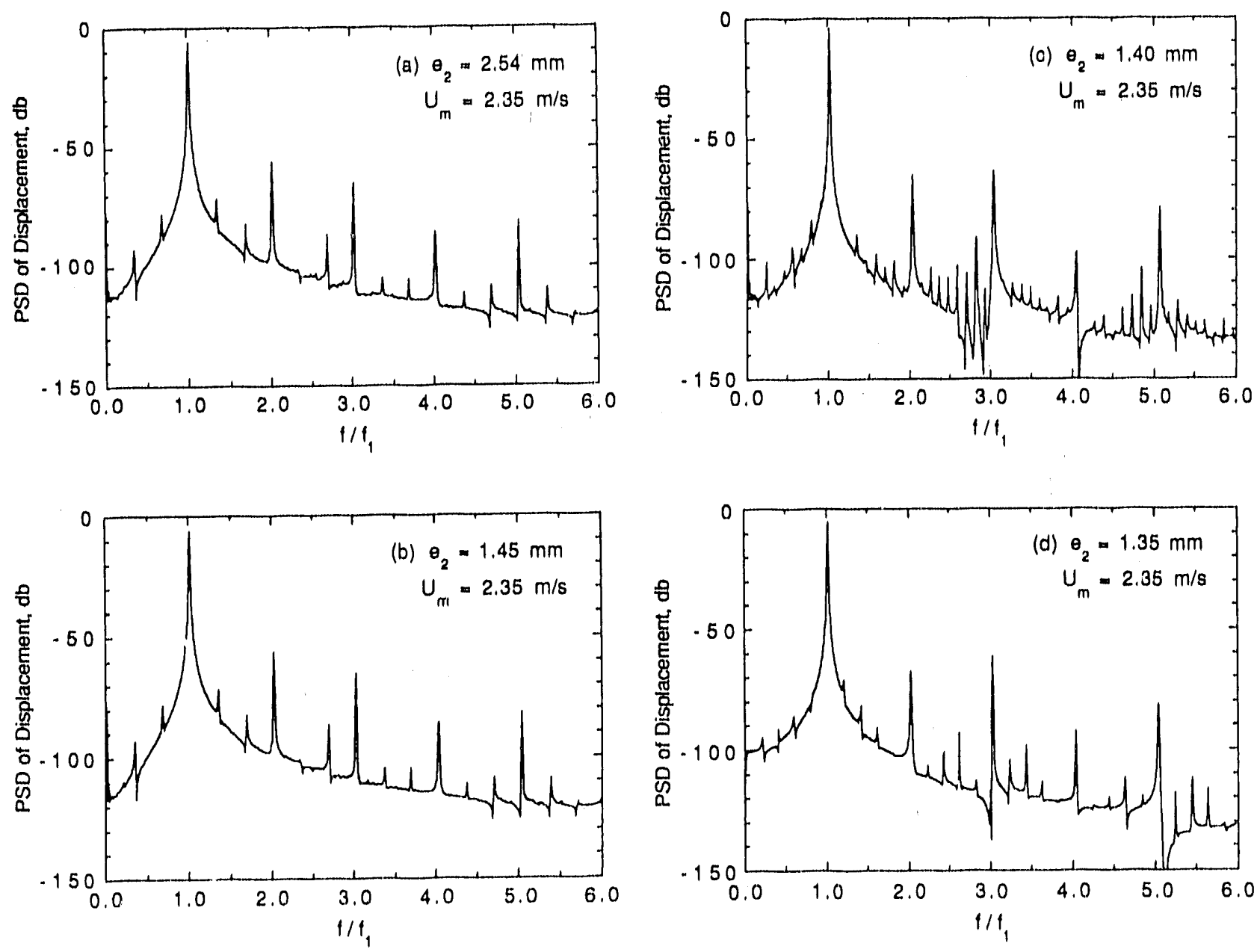

Fig. 16. PSD of tube motion with $U_{m}=2.35 \mathrm{~m} / \mathrm{s}$ and asymmetric clearances $e_{1}=1.27 \mathrm{~mm}$ and (a) $e_{2}=2.54 \mathrm{~mm}$, (b) $e_{2}=1.45 \mathrm{~mm},(c) e_{2}=1.40 \mathrm{~mm}$, and (d) $e_{2}=1.35 \mathrm{~mm}$

As suggested in most investigations on chaotic motion (Moon, 1987), it is important to use more than one measurement in deciding on the existence of chaos. Thus, we carried out many measurements in this study, including those with phase flow portraits, PSD, Poincaré maps, Lyapunov exponents, fractal dimension, and bifurcation diagrams, to confirm the existence of chaotic motion in the instability region. Fortunately, the measured results with those effective tools were in perfect agreement to convincingly demonstrate that in a certain flow velocity range, chaotic motions do arise: the phase portrait displays a band circle to indicate limited-band chaotic motion; PSD presents a continuous limited-band spectrum; the Poincaré map gives a scattered structure; Lyapunov exponents appear to be positive; and the fractal dimension is $\approx 1.55$ (not an integer). The importance of this, to our best knowledge, is that this is the first application of so many measurements that give consistent results in exploring the chaotic motion of loosely supported tubes that is induced by fluidelastic instability in crossflow. 

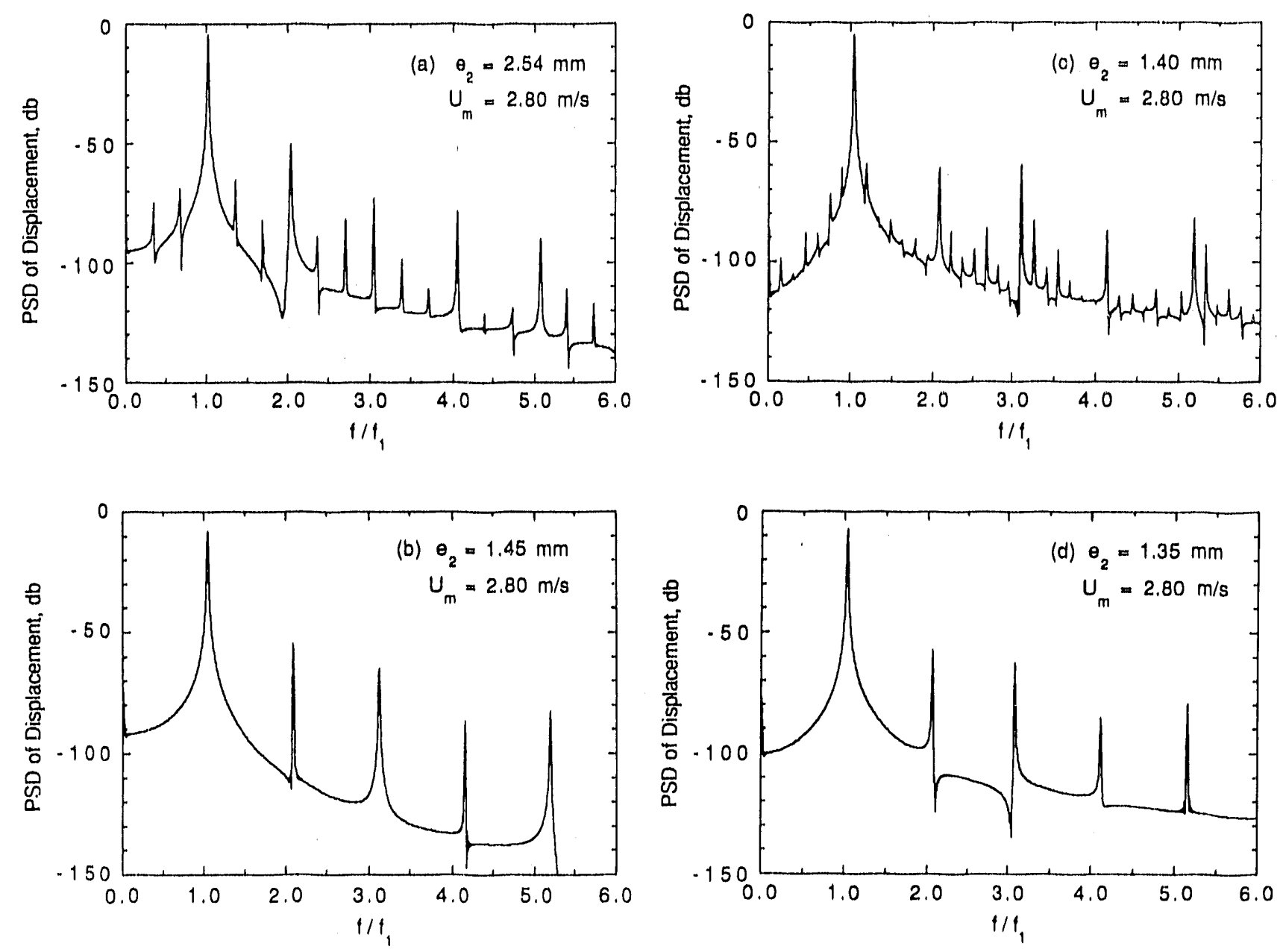

Fig. 17. PSD of tube motion with $U_{m}=2.80 \mathrm{~m} / \mathrm{s}$ and asymmetric clearances $e_{1}=1.27 \mathrm{~mm}$ and (a) $e_{2}=2.54 \mathrm{~mm}$, (b) $e_{2}=1.45 \mathrm{~mm}$, (c) $e_{2}=1.40 \mathrm{~mm}$, and (d) $e_{2}=1.35 \mathrm{~mm}$

The bifurcation diagrams show the route of chaotic-periodic-chaotic motion in the instability region corresponding to the variations of negative damping versus mean flow velocity. It is very important that the distribution of chaotic/periodic motions depends on the negative damping of both Models 1 and 2. When damping varies, the number of the tube strikes with the TSP changes. If the tube strikes the TSP regularly, it is periodic (or quasiperiodic) motion; if the tube strikes the TSP irregularly, chaotic motion may develop. This reminds us to pay more attention to the striking situation when predicting chaotic/periodic motions in further research. Also, we note that nonlinearity of the TSP, with symmetric or asymmetric gaps, significantly affects the distribution of periodic, quasiperiodic, and chaotic motion of the tube with varying flow velocity in the instability region of the TSP-inactive mode. Chaotic motion will be continuously subjected to parameter analysis to explore its behavior in more detail. 
In this study, a very complicated system, i.e., a tube with two asymmetric spans subjected to nonuniform crossflow, was analyzed in 10 modes. Therefore, the chaotic behavior of the system may be quite different from systems with one or two degrees of freedom, as often investigated by researchers exploring chaotic motions. Even though the analytical results in our studies demonstrated the existence and identified some characteristics of chaotic motion in the instability region of the TSP-inactive mode, we still have difficulties in completely understanding why the chaotic motion occurs and how we can control it economically.

Obviously, further research is needed on the effects of chaotic motion on the fluidelastic instability of loosely supported tubes. A test is in progress at Argonne National Laboratory to obtain experimental data on periodic/chaotic motion of a loosely supported tube in crossflow. Detailed comparisons of the analytical and experimental results will be published when testing is completed. This will bring us more information on, and evidence of, the effects of chaos on the fluidelastic instability of a loosely supported tube in crossflow.

\section{Acknowledgments}

This work was sponsored by the U.S. Department of Energy, Office of Basic Energy Sciences, Division of Engineering and Geosciences, under Contract W-31109-Eng-38.

\section{References}

Antunes, J., Axisa, F., and Vento, M. A., 1991, Experiments on Vibro-Impact Dynamics Under Fluidelastic Instability, ASME Pressure Vessels and Piping Conf., Nashville, TN, PVP Vol. 189, pp. 127-138.

Axisa, F., Antunes, J., and Villard, B., 1988, Overview of Numerical Methods for Predicting Flow-Induced Vibration, Trans. ASME Pressure Vessels and Piping, Vol. 110, pp. 6-14.

Baker, G. L., and Gollub, J. P., 1990, Chaotic Dynamics, New York: Cambridge University Press.

Cai, Y., and Chen, S. S., 1991, Chaotic Dynamics of Loosely Supported Tubes in Crossflow, Argonne National Laboratory Report ANL-91/30 (July). 
Cai, Y., Chen, S. S., and Chandra, S., 1991, A Theory for Fluidelastic Instability of Tube-Support-Plate Inactive Modes, ASME Pressure Vessels and Piping Conf., San Diego, CA, PVP-Vol, 206, pp. 9-18; To appear in Journal of Pressure Vessel Technology.

Chen, S. S., 1983, Instability Mechanism and Stability Criteria of a Group of Circular Cylinders Subjected to Crossflow, Part 1: Theory, J. Vibration, Acoustics, Stress and Reliability in Design, Vol. 105, pp. 51-58.

Chen, S. S., 1989, Some Issues Concerning Fluidelastic Instability of a Group of Circular Cylinders in Cross-Flow, Trans. ASME, J. Pressure Vessel Technol., Vol. 111, pp. 507-518.

Chen, S. S., 1991, A Review of Dynamic Tube-Support Interaction in Heat Exchanger Tubes, Presented at the Fifth Int. Conf. on Flow Induced Vibrations, Brighton, England, Paper C416/012.

Chen, S. S., and Chandra, S., 1990, Fluidelastic Instabilities in Tube Bundles Exposed to Nonuniform Crossflow, ASME Pressure Vessels and Piping Conf., Nashville, TN, PVP Vol. 189, pp. 65-77.

Chen, S. S., Jendrzejczyk, J. A., and Wambsganss, M. W., 1984, Dynamics of Tubes in Fluid with Tube-Baffle Interaction, ASME Symp. on Flow-Induced Vibration, New Orleans, Vol. 2, pp. 285-304.

Dowell, E. H., 1984, Observation and Evolution of Chaos for an Autonomous System, J. Appl. Mech., Vol. 51, pp. 333-344.

Fisher, N. J., and Ingham, B., 1988, Measurement of Tube-to-Support Dynamic Force in Fretting-Wear Rigs, Int. Symp. on Flow-Induced Vibration and Noise, Chicago, Vol. 5, pp. 137-156.

Fricker, A. J., 1988, Numerical Analysis of the Fluidelastic Vibration of a Steam Generator Tube with Loose Supports, Int. Symp. on Flow-Induced Vibration and Noise, Chicago, Vol. 5, pp. 105-120.

Grassberger, P., and Proccacia, J., 1983, Measuring the Strangeness of Strange Attractors, Physica, Vol. 9D, 189-208.

Langre, E. de, et al., 1990, Chaotic and Periodic Motion of a Non-Linear Oscillator in Relation with Flow-Induced Vibrations of Loosely Supported Tubes, ASME Pressure Vessels and Piping Conf., Nashville, TN, PVP Vol. 189, pp. 119125. 
Moon, F. C., 1987, Chaotic Vibrations, New York: John Wiley.

Moon, F. C., and Shaw, S. W., 1983, Chaotic Vibrations of a Beam with NonLinear Boundary Conditions, Int. J. Non-Linear Mechanics, Vol. 18, pp. 465-477.

Nakamura, T., and Fujita, K., 1987, A Study on Impact Vibration of Loosely Held Tube by Cross Flow, Int. Conf. on Flow Induced Vibrations, Bowness-onWindermere, England, Faper K1, pp. 427-436.

Paidoussis, M. P., and Moon, F. C., 1988, Nonlinear and Chaotic Fluidelastic Vibrations of a Flexible Pipe Conveying Fluid, J. Fluids and Structures, Vol. 2, pp. 567-591.

Paidoussis, M. P., and Moon, F. C., 1989, Chaotic Oscillations of the Autonomous System of a Constrained Pipe Conveying Fluid, J. Sound Vib., Vol. 135(1), pp. 1-19.

Rao, M. S. M., Steininger, D. A., and Eisinger, F. L., 1988, Numerical Simulation of Fluidelastic Vibration and Wear of Multispan Tubes with Clearances at Supports, Int. Symp. on Flow-Induced Vibration and Noise, Chicago, Vol. 5, pp. 235-250.

Shaw, S. W., 1985a, The Dynamics of a Harmonically Excited System Having Rigid Amplitude Constraints; Parts I and II, ASME J. Appl. Mech., Vol. 52, pp. 453-458 and 459-469.

Shaw, S. W., 1985b, Forced Vibrations of a Beam with One-Sided Amplitude Constraint: Theory and Experiment, J. Sound Vib., Vol. 99, pp. 199-212.

Shaw, S. W., and Holmes, P. J., 1983, A Periodically Forced Piecewise Linear Oscillator, J. Sound Vib., Vol. 90, pp. 129-155.

Tanaka, M., 1980, Study on Fluidelastic Vibrations of Tube Bundle, Jpn. Soc. Mech. Eng., Trans., Section B, Vol. 46(408), pp. 1398-1407.

Thompson, J. M. T., and Stewart, H. B., 1986, Nonlinear Dynamics and Chaos, New York: John Wiley.

Wolf, A., Swift, J. B., Swinney, H. L., and Vastano, J. A., 1985, Determining Lyapunov Exponents from a Time Series, Physics, Vol. 16D, 285-317. 


\section{Appendix: \\ Modal Analysis of a Bllinear Model}

We assume that during the time interval $0 \leq \mathrm{t} \leq \mathrm{t}_{\mathrm{s}}$, the tube displacements at C3 are within the stop limits $-e_{2}<u(1, t)<e_{1}$, and it is Model 1. The boundary conditions at $\mathrm{C} 1$ and $\mathrm{C} 3$ (see Fig. 1) are

$$
\begin{aligned}
& \left.u(\xi, t)\right|_{\xi=0}=0, \\
& \left.\frac{\partial^{2} u}{\partial \xi^{2}}(\xi, t)\right|_{\xi=0}=0, \\
& \left.\frac{\partial^{2} u}{\partial \xi^{2}}(\xi, t)\right|_{\xi=1}=0, \\
& \left.\frac{\partial^{3} u}{\partial \xi^{3}}(\xi, t)\right|_{\xi=1}=0,
\end{aligned}
$$

and the continuous conditions at the intermediate point $\mathrm{C} 2$ are

$$
\begin{aligned}
& \left.\mathrm{u}(\xi, \mathrm{t})\right|_{\xi=\mu}=0, \\
& \left.\frac{\mathrm{d} \varphi_{1 \mathrm{n}}^{(1)}(\xi)}{\mathrm{d} \xi}\right|_{\xi=\mu}=\left.\frac{\mathrm{d} \varphi_{1 \mathrm{n}}^{(2)}(\xi)}{\mathrm{d} \xi}\right|_{\xi=\nu}, \\
& \left.\frac{\mathrm{d}^{2} \varphi_{1 \mathrm{n}}^{(1)}(\xi)}{\mathrm{d} \xi}\right|_{\xi=\mu}=-\left.\frac{\mathrm{d}^{2} \varphi_{\ln }^{(2)}(\xi)}{\mathrm{d} \xi}\right|_{\xi=\nu},
\end{aligned}
$$

where

$$
\begin{aligned}
& \xi=z / \ell, \\
& \mu=\ell_{1} / \ell, \\
& v=\ell_{2} / \ell=1-\mu .
\end{aligned}
$$

When $t=t_{s}$, the right end of the tube strikes the stop and it becomes Model 2, i.e., 


$$
\left.\mathbf{u}(\xi, \mathrm{t})\right|_{\xi=1}=e_{1}
$$

or

$$
\begin{aligned}
& \left.\mathrm{u}(\xi, \mathrm{t})\right|_{\xi=1}=-\mathrm{e}_{2}, \\
& \mathrm{t}_{\mathrm{B}}<\mathrm{t}<\mathrm{t}_{\mathrm{d}},
\end{aligned}
$$

where $t_{d}$ is the time when the tube leaves the stop at C3.

The boundary conditions of Model 2 for $t_{\mathrm{s}}<t<t_{d}$ are

$$
\begin{aligned}
& \left.u(\xi, t)\right|_{\xi=0}=0, \\
& \left.\frac{\partial^{2} u}{\partial \xi^{2}}(\xi, t)\right|_{\xi=0}=0, \\
& \left.\frac{\partial^{2} u}{\partial \xi^{2}}(\xi, t)\right|_{\xi=1}=0, \\
& \left.\frac{\partial^{3} u}{\partial \xi^{3}}(\xi, t)\right|_{\xi=1}=\left.\frac{K_{c}}{E I} u(\xi, t)\right|_{\xi=1}
\end{aligned}
$$

where $K_{c}$ is the equivalent stiffness, $E$ is Young's modulus, and $I$ is the moment of inertia.

The continuous conditions at the intermediate point $\mathrm{C} 2$ are

$$
\begin{aligned}
& \left.\mathrm{u}(\xi, \mathrm{t})\right|_{\xi=\mu}=0, \\
& \left.\frac{\mathrm{d} \varphi_{2 n}^{(1)}(\xi)}{\mathrm{d} \xi}\right|_{\xi=\mu}=\left.\frac{\mathrm{d} \varphi_{2 \mathrm{n}}^{(2)}(\xi)}{\mathrm{d} \xi}\right|_{\xi=\nu}, \\
& \left.\frac{\mathrm{d}^{2} \varphi_{2 \mathrm{n}}^{(1)}(\xi)}{\mathrm{d} \xi}\right|_{\xi=\mu}=-\left.\frac{\mathrm{d}^{2} \varphi_{2 n}^{(2)}(\xi)}{\mathrm{d} \xi}\right|_{\xi=\nu} .
\end{aligned}
$$


$\varphi_{\text {in }}^{(1)}(\xi)$ and $\varphi_{\text {in }}^{(2)}(\xi)(\mathrm{i}=1,2)$ are the normal modes $f$ " Wi.odels 1 and 2.

$$
\begin{aligned}
& \varphi_{\text {in }}^{(1)}(\xi)=\sin k_{\text {in }} \xi-\frac{\sin k_{\text {in }} \mu}{\operatorname{sh} k_{\text {in }} \mu} \operatorname{sh} k_{\text {in }} \xi, \\
& 0<\xi<\mu, \quad \mathrm{i}=1,2, \quad \mathrm{n}=1,2,3, \ldots \\
& \varphi_{\text {in }}^{(2)}(\xi)=D_{1}\left\{D _ { 2 } \left[\cos k_{\text {in }}\left(1-\xi j+\operatorname{ch} k_{\text {in }}(1-\xi)\right]+D_{3}\left[\sin k_{\text {in }}(1-\xi)+\operatorname{sh} k_{\text {in }}(1-\xi)\right]\right.\right. \\
& \left.+D_{4}\left[D_{5} \sin k_{\text {in }}(1-\xi)+D_{6} \operatorname{sh~} k_{\text {in }}(1-\xi)\right]\right\} \text {, } \\
& \mu<\xi<1, \quad i=1,2, \quad \mathrm{n}=1,2,3, \ldots,
\end{aligned}
$$

where

$$
\begin{aligned}
& D_{1}=\frac{\sin k_{\text {in }} \mu}{\cos k_{\text {in }} v \operatorname{sh} k_{\text {in }} v-\sin k_{\text {in }} v \operatorname{ch} k_{\text {in }} v-\frac{2 K}{E I k_{\text {in }}^{3}} \sin k_{\text {in }} v \operatorname{sh} k_{\text {in }} v}, \\
& D_{2}=\sin k_{\text {in }} v+\operatorname{sh} k_{\text {in }} \nu \text {, } \\
& \mathrm{D}_{3}=-\cos \mathrm{k}_{\mathrm{in}} v-\operatorname{ch} \mathrm{k}_{\mathrm{in}} v \text {, } \\
& \mathrm{D}_{4}=\frac{2 \mathrm{~K}}{\mathrm{EI} \mathrm{k}_{\mathrm{in}}^{3}} \\
& D_{5}=-s h k_{i n} v \text {, } \\
& \mathrm{D}_{6}=\sin \mathrm{k}_{\mathrm{in}} v, \\
& K= \begin{cases}0 & \text { for } i=1 \\
K_{c} & \text { for } i=2 .\end{cases}
\end{aligned}
$$

The eigenvalues $k_{\text {in }}$ are the solutions of the characteristic equation 


$$
\begin{aligned}
& \frac{\sin k_{\text {in }} \mu}{\cos k_{\text {in }} \mu-\sin k_{\text {in }} \mu \operatorname{cth} k_{\text {in }} \mu} \\
& -\frac{\cos k_{\text {in }} v \operatorname{sh~} k_{\text {in }} \nu-\sin k_{\text {in }} v \operatorname{ch~} k_{\text {in }} v-\frac{2 K}{E I k_{\text {in }}^{3}} \sin k_{\text {in }} v \operatorname{sh~} k_{\text {in }} v}{2\left(1+\cos k_{\text {in }} v \operatorname{ch~} k_{\text {in }} v\right)+\frac{2 K}{E I k_{\text {in }}^{3}}\left(\cos k_{\text {in }} v \operatorname{sh~} k_{\text {in }} v-\sin k_{\text {in }} v \operatorname{ch~} k_{\text {in }} v\right)}=0 .
\end{aligned}
$$

The eigenvalues of the first 10 modes for both Models 1 and 2, with tube parameters shown in Fig, 1 and the impact stiffness of springs at the stops $K_{c}=$ $10^{7} \mathrm{~N} / \mathrm{m}$, are given in Table A.1.

Table A.1. Eigenvalues $k_{\text {in }}$ of first 10 modes for Models 1 and 2

\begin{tabular}{crr}
\hline $\mathrm{n}$ & $\mathrm{i}=1$ & $\mathrm{i}=2$ \\
\hline 1 & 3.92 & 4.89 \\
2 & 6.10 & 8.90 \\
3 & 9.15 & 12.57 \\
4 & 13.06 & 14.39 \\
5 & 16.25 & 21.56 \\
6 & 21.72 & 25.19 \\
7 & 25.68 & 27.02 \\
8 & 28.71 & 30.26 \\
9 & 30.63 & 34.24 \\
10 & 34.37 & 37.92 \\
\hline
\end{tabular}

Let

$$
\begin{aligned}
& \varphi_{\text {in }}(\xi)= \begin{cases}\mathrm{C}_{\mathrm{in}} \varphi_{\text {in }}^{(1)}(\xi) & 0<\xi<\mu \\
\mathrm{C}_{\text {in }} \varphi_{\text {in }}^{(2)}(\xi) & \mu<\xi<1,\end{cases} \\
& \mathrm{i}=1,2, \quad \mathrm{n}=1,2,3, \ldots
\end{aligned}
$$

where 


$$
\mathrm{C}_{\mathrm{in}}=\frac{1}{\sqrt{\int_{0}^{\mu}\left[\varphi_{\mathrm{in}}^{(1)}(\xi)\right]^{2} \mathrm{~d} \xi+\int_{\mu}^{1}\left[\varphi_{\mathrm{in}}^{(2)}(\xi)\right]^{2} \mathrm{~d} \xi}}
$$

The solutions of the tube vibration by the normal-mode method are

$$
\left.\begin{array}{l}
u(\xi, t)=\sum_{n=1}^{\infty} a_{1 n}(t) \varphi_{1 n}(\xi) \\
\dot{u}(\xi, t)=\sum_{n=1}^{\infty} \dot{c}_{i 1}(t) \varphi_{\ln }(\xi)
\end{array}\right\} \quad 0<t<t_{s} \text { for Model } 1
$$

and

$$
\begin{aligned}
& u(\xi, t)=\sum_{n=1}^{\infty} a_{2 n}(t) \varphi_{2 n}(\xi)+\left.u(\xi, t)\right|_{t=t_{s}} \\
& \dot{\mathrm{u}}(\xi, \mathrm{t})=\sum_{\mathrm{n}=1}^{\infty} \dot{\mathrm{a}}_{2 \mathrm{n}}(\mathrm{t}) \varphi_{2 \mathrm{n}}(\xi) \\
& t_{s}<t<t_{d} \text { for Model 2, }
\end{aligned}
$$

where $a_{\text {in }}$ and $\dot{a}_{\text {in }}(t)$ are the normal coordinates, which are the solutions of following equations:

$$
\begin{aligned}
& \left(1+\gamma \alpha_{\text {in }}\right) \frac{\mathrm{d}^{2} \mathrm{a}_{\text {in }}}{d \mathrm{dt}^{2}}+\left(2 \zeta_{\mathrm{n}} \omega_{\text {in }}-\frac{\gamma \mathrm{U}_{\mathrm{r}}^{2}}{\pi^{3}} \omega_{\mathrm{i}} \alpha_{\mathrm{in}}^{\mathrm{d}}\right) \frac{\mathrm{da} \mathrm{a}_{\text {in }}}{\mathrm{dt}}+\left(\omega_{\text {in }}^{2}-\frac{\gamma \mathrm{U}_{\mathrm{r}}^{2}}{\pi^{3}} \alpha_{\mathrm{in}}^{\mathrm{e}}\right) \mathrm{a}_{\mathrm{in}}=0, \\
& \mathrm{i}=1,2, \quad \mathrm{n}=1,2,3, \ldots
\end{aligned}
$$

and the initial conditions:

$$
\left.\begin{array}{l}
\mathrm{a}_{1 \mathrm{n}}(0)=\int_{0}^{1}\left[\left.\mathrm{u}(\xi, \mathrm{t})\right|_{\mathrm{t}=0}\right] \varphi_{1 \mathrm{n}}(\xi) \mathrm{d} \xi, \\
\dot{\mathrm{a}}_{1 \mathrm{n}}(0)=\int_{0}^{1}\left[\left.\dot{\mathrm{u}}(\xi, \mathrm{t})\right|_{\mathrm{t}=0}\right] \varphi_{1 \mathrm{n}}(\xi) \mathrm{d} \xi,
\end{array}\right\}
$$


34

and

$$
\left.\begin{array}{l}
\mathbf{a}_{2 \mathrm{n}}(0)=0, \\
\dot{\mathrm{a}}_{2 \mathrm{n}}(0)=\int_{0}^{1}\left[\left.\dot{\mathrm{u}}(\xi, t)\right|_{t=t_{\mathrm{s}}}\right] \varphi_{2 \mathrm{n}}(\xi) \mathrm{d} \xi .
\end{array}\right\}
$$

(A16) 


\section{Distribution for ANL-92/18}

\section{Internal}

Y. Cai (10)

S. S. Chen (10)

S. H. Chen

J. A. Jendrzejczyk

C. A. Malefyt (2)

T. M. Mulcahy

C. E. Till
R. A. Valentin

M. W. Wambsganss (3)

R. W. Weeks

S. Zhu

ANL Patent Dept.

ANL Contract File

TIS Files (3)

\section{External}

DOE-OSTI for distribution per UC-406 (47)

ANL-TIS Libraries (2)

Manager, Chicago Operations Office, DOE

Director, Technology Management Div., DOE-CH

D. L. Bray, DOE-CH

A. L. Taboas, DOE-CH

Materials and Components Technology Division Review Committee:

H. Berger, Industrial Quality, Inc., Gaithersburg, MD

M. S. Dresselhaus, Massachusetts Institute of Technology, Cambridge, MA

S. J. Green, Electric Power Research Institute, Palo Alto, CA

R. A. Greenkorn, Purdue University, West Lafayette, IN

C.-Y. Li, Cornell University, Ithaca, NY

P. G. Shewmon, Ohio State University, Columbus

R. E. Smith, Electric Power Research Institute, NDE Ctr., Charlotte, NC

O. P. Manley, DOE, Washington, DC

J. S. Coleman, DOE, Washington, DC

D. Frederick, DOE, Washington, DC

F. C. Moon, Cornell University, Ithaca, NY 

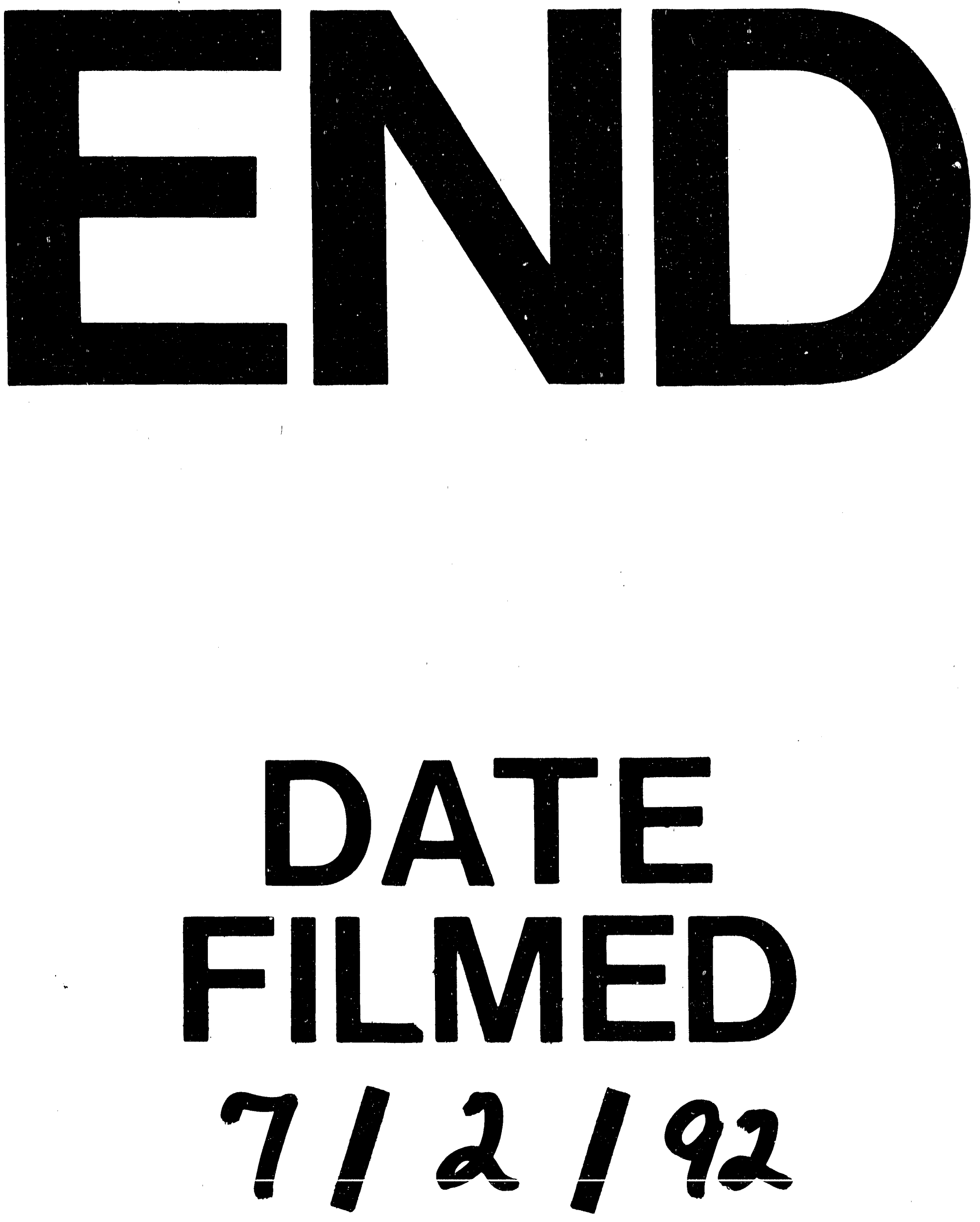
